\title{
A Month of Monitoring the New Magnetar Swift J1555.2-5402 during an X-Ray Outburst
}

\author{
Teruaki Enoto $^{1}$ (iD), Mason $\mathrm{Ng}^{2}$ (D) ${\text {, Chin-Ping } \mathrm{Hu}^{3} \text { (D), Tolga Güver }}^{4,5}$ (iD), Gaurava K. Jaisawal ${ }^{6}$ (i), Brendan O’Connor ${ }^{7,8,9,10}$ (iD), \\ Ersin Göğüş $^{11}$ (D), Amy Lien ${ }^{12,13,14}$, Shota Kisaka $^{15}$ (D), Zorawar Wadiasingh ${ }^{10,16}$ (D), Walid A. Majid ${ }^{17,18}$ (iD), \\ Aaron B. Pearlman ${ }^{18,19,20,31,32}$ (D), Zaven Arzoumanian ${ }^{10}$, Karishma Bansal $^{17}$ (D), Harsha Blumer ${ }^{21,22}$ (D), Deepto Chakrabarty ${ }^{2}$ (D), \\ Keith Gendreau $^{10}$ (D), Wynn C. G. Ho ${ }^{23}$ (D), Chryssa Kouveliotou ${ }^{7,8}$ (D), Paul S. Ray ${ }^{24}$ (D), Tod E. Strohmayer ${ }^{25}$ (D), \\ George Younes $^{7,8}$ (D), David M. Palmer ${ }^{26}$ (D), Takanori Sakamoto ${ }^{27}$ (D), Takuya Akahori ${ }^{28,29}$ (D), and Sujin Eie ${ }^{28,30}$ (D) \\ ${ }^{1}$ RIKEN Cluster for Pioneering Research, 2-1 Hirosawa, Wako, Saitama 351-0198, Japan \\ ${ }^{2}$ MIT Kavli Institute for Astrophysics and Space Research, Massachusetts Institute of Technology, Cambridge, MA 02139, USA \\ ${ }^{3}$ Department of Physics, National Changhua University of Education, Changhua 50007, Taiwan \\ ${ }^{4}$ Istanbul University, Science Faculty, Department of Astronomy and Space Sciences, Beyazit, 34119, İstanbul, Turkey \\ ${ }^{5}$ Istanbul University Observatory Research and Application Center, Istanbul University 34119, İstanbul, Turkey \\ ${ }^{6}$ National Space Institute, Technical University of Denmark, Elektrovej 327-328, DK-2800 Lyngby, Denmark \\ ${ }^{7}$ Department of Physics, The George Washington University, 725 21st Street NW, Washington, DC 20052, USA \\ ${ }^{8}$ Astronomy, Physics and Statistics Institute of Sciences (APSIS), The George Washington University, Washington, DC 20052, USA \\ ${ }^{9}$ Department of Astronomy, University of Maryland, College Park, MD 20742-4111, USA \\ ${ }^{10}$ Astrophysics Science Division, NASA Goddard Space Flight Center, Greenbelt, MD 20771, USA \\ ${ }^{11}$ Sabanc1 University, Faculty of Engineering and Natural Sciences, İstanbul 34956, Turkey
${ }^{12}$ Center for Research and Exploration in Space Science and Technology (CRESST) and NASA Goddard Space Flight Center, Greenbelt, MD 20771, USA \\ ${ }^{13}$ Department of Physics, University of Maryland, Baltimore County, 1000 Hilltop Circle, Baltimore, MD 21250, USA \\ ${ }^{14}$ University of Tampa, Department of Chemistry, Biochemistry, and Physics, 401 West Kennedy Boulevard, Tampa, FL 33606, USA \\ 5 Department of Physics, Hiroshima University, Higashi-Hiroshima, 739-8526, Japan \\ ${ }^{16}$ Universities Space Research Association (USRA) Columbia, MD 21046, USA \\ ${ }^{17}$ Jet Propulsion Laboratory, California Institute of Technology, Pasadena, CA 91109, USA \\ ${ }^{18}$ Division of Physics, Mathematics, and Astronomy, California Institute of Technology, Pasadena, CA 91125, USA \\ ${ }_{19}$ Department of Physics, McGill University, 3600 rue University, Montréal, QC H3A 2T8, Canada \\ ${ }_{20}^{20}$ McGill Space Institute, McGill University, 3550 rue University, Montréal, QC H3A 2A7, Canada \\ ${ }^{21}$ Department of Physics and Astronomy, West Virginia University, Morgantown, WV 26506, USA \\ ${ }^{22}$ Center for Gravitational Waves and Cosmology, West Virginia University, Chestnut Ridge Research Building, Morgantown, WV 26505, USA \\ ${ }^{23}$ Department of Physics and Astronomy, Haverford College, 370 Lancaster Avenue, Haverford, PA 19041, USA \\ ${ }^{24}$ Space Science Division, U.S. Naval Research Laboratory, Washington, DC 20375, USA \\ ${ }^{25}$ Astrophysics Science Division and Joint Space-Science Institute, NASA's Goddard Space Flight Center, Greenbelt, MD 20771, USA \\ ${ }^{26}$ Los Alamos National Laboratory, Los Alamos, NM 87545, USA \\ ${ }^{27}$ College of Science and Engineering, Department of Physical Sciences, Aoyama Gakuin University, 5-10-1 Fuchinobe, Chuo-ku, Sagamihara-shi Kanagawa 252- \\ 5258, Japan \\ ${ }^{28}$ Mizusawa VLBI Observatory, National Astronomical Observatory of Japan, 2-21-1, Osawa, Mitaka, Tokyo 181-8588, Japan \\ ${ }^{29}$ Operation Division, Square Kilometre Array Observatory, Jodrel Bank Observatory, Lower Withington, Macclesfield, Cheshire SK11 9FT, UK \\ ${ }^{30}$ Department of Astronomy, Graduate School of Science, The University of Tokyo, 7-3-1 Hongo, Bunkyo-ku, Tokyo 113-0033, Japan \\ Received 2021 August 5; revised 2021 September 8; accepted 2021 September 10; published 2021 October 5
}

\begin{abstract}
The soft gamma-ray repeater Swift J1555.2-5402 was discovered by means of a short burst detected with Swift BAT on 2021 June 3. Then, $1.6 \mathrm{hr}$ after the burst, the Neutron star Interior Composition Explorer (NICER) started daily monitoring of this target for a month. The absorbed $2-10 \mathrm{keV}$ flux stayed nearly constant at around $4 \times 10^{-11} \mathrm{erg} \mathrm{s}^{-1} \mathrm{~cm}^{-2}$ during the monitoring, showing only a slight gradual decline. An absorbed blackbody with a temperature of $1.1 \mathrm{keV}$ approximates the soft X-ray spectrum. A $3.86 \mathrm{~s}$ periodicity is detected, and the period derivative is measured to be $3.05(7) \times 10^{-11} \mathrm{~s} \mathrm{~s}^{-1}$. The soft $\mathrm{X}$-ray pulse shows a single sinusoidal shape with an rms pulsed fraction that increases as a function of energy from $15 \%$ at $1.5 \mathrm{keV}$ to $39 \%$ at $7 \mathrm{keV}$. The equatorial surface magnetic field, characteristic age, and spin-down luminosity are derived under the dipole field approximation to be $3.5 \times 10^{14} \mathrm{G}, 2.0 \mathrm{kyr}$, and $2.1 \times 10^{34} \mathrm{erg} \mathrm{s}^{-1}$, respectively. We detect 5 and 45 bursts with Swift/BAT and NICER, respectively. Based on these properties, this new source is classified as a magnetar. A hard X-ray power-law component that extends up to at least $40 \mathrm{keV}$ is detected with the Nuclear Spectroscopic Telescope Array (NuSTAR). The $10-60 \mathrm{keV}$ flux is $\sim 9 \times 10^{-12} \mathrm{erg} \mathrm{s}^{-1} \mathrm{~cm}^{-2}$ with a photon index of $\sim 1.2$. The pulsed fraction has a sharp cutoff at around $10 \mathrm{keV}$ with an upper limit $(\lesssim 10 \%)$ in the hard-tail band. No radio pulsations are detected during the DSN or VERA observations. The $7 \sigma$ upper limits of the flux density are 0.043 and $0.026 \mathrm{mJy}$ at the $S$ and $X$ bands, respectively.
\end{abstract}

Unified Astronomy Thesaurus concepts: Magnetars (992); Magnetic fields (994); Neutron stars (1108); Pulsars (1306); Soft gamma-ray repeaters (1471); X-ray transient sources (1852)

\footnotetext{
31 McGill Space Institute (MSI) Fellow.

${ }^{32}$ FRQNT Postdoctoral Fellow.
}

\section{Introduction}

Magnetars are highly magnetized neutron stars that are usually bright in X-rays as a result of the release of an 
enormous amount of magnetic energy stored in the stellar interior and the magnetosphere (Mereghetti 2008; Kaspi \& Beloborodov 2017). Among them, sources emitting repetitive soft gamma-ray bursts are historically called soft gamma-ray repeaters (SGRs; e.g., Kouveliotou et al. 1998). In the last decade, systematic monitoring of magnetars in X-rays, mainly with the X-Ray Telescope (XRT) on board the Neil Gehrels Swift Observatory, revealed that many transient magnetars spend most of their time in a quiescent state with low activity. However, they occasionally exhibit a sudden X-ray brightening, where the X-ray flux reaches an initial plateau of $10^{-11}-10^{-10} \mathrm{erg} \mathrm{s}^{-1} \mathrm{~cm}^{-2}$ lasting a few weeks, followed by a gradual decay over a variety of timescales (Enoto et al. 2017; Coti Zelati et al. 2018). These magnetar outbursts are characterized by enhanced persistent X-ray emission, sporadic short bursts, pulsar timing anomalies, and, rarely, giant flares. Their origin has been attributed to various mechanisms, such as the relaxation process of twisted magnetic fields, starquakes, magnetothermal evolution, and magnetic field dissipation (Thompson \& Duncan 1995, 1996; Perna \& Pons 2011; Beloborodov \& Li 2016). Multiwavelength observations of these outbursts are essential to address a wide range of astronomical topics, as demonstrated, for example, with the discovery of fast radio bursts (FRBs) associated with a short hard X-ray burst from the Galactic magnetar SGR J1935+2154 in 2020 (Bochenek et al. 2020; CHIME/FRB Collaboration et al. 2020; Mereghetti et al. 2020; Li et al. 2021; Ridnaia et al. 2021; Tavani et al. 2021). Such a connection between magnetars and FRBs is also supported by the indication that extragalactic FRBs have the statistical signatures of magnetar short bursts (e.g., Wadiasingh \& Timokhin 2019).

On 2021 June 3, a new SGR, Swift J1555.2-5402, was discovered through a short burst detection with the Burst Alert Telescope (BAT) on board Swift (Palmer et al. 2021). Immediately after the notification of the burst from this source, several X-ray satellites started follow-up observations of this magnetar candidate. These observations were promptly used to measure the spin frequency and frequency derivative (Israel et al. 2021; Ng et al. 2021; Coti Zelati et al. 2021b) and detected several short bursts (Palmer 2021). Based on the measured timing parameters and distinctive magnetar characteristics, this new source was classified as a magnetar. In addition, several radio telescopes searched for radio emission and pulsations (Bansal et al. 2021; Burgay et al. 2021; Singh \& Roy 2021). In this Letter, we report on the X-ray temporal and spectral characteristics of this new magnetar observed with Swift, the Neutron star Interior Composition Explorer (NICER), and the Nuclear Spectroscopic Telescope Array (NuSTAR) during the initial 29 days of its X-ray outburst; our observations were also coordinated with radio monitoring.

The celestial position of Swift J1555.2-5402 is close to another magnetar, 1E 1547.0-5408, with an angular separation of about 0.7 . The distance to $1 \mathrm{E} 1547.0-5408$ is estimated to be $4-4.5 \mathrm{kpc}$ from a dust-scattering halo (Tiengo et al. 2010) and a possible association with SNR G327.24-0.13 (Gelfand $\&$ Gaensler 2007). By contrast, no definitive information is available about the distance to Swift J1555.2-5402 (Section 3.5). Taking into account the fact that the column density of Swift J1555.2-5402 $\left(N_{\mathrm{H}}=8.7 \times 10^{22} \mathrm{~cm}^{-2}\right)$ is larger than that of $1 \mathrm{E} 1547.0-5408\left(N_{\mathrm{H}}=3.2 \times 10^{22} \mathrm{~cm}^{-2}\right.$; Enoto et al. 2010b), we assume a fiducial distance $d$ of $10 \mathrm{kpc}$ and the normalization factor $d_{10}=d /(10 \mathrm{kpc})$ throughout this
Letter. This distance corresponds to a location on the ScutumCentaurus Arm.

\section{Observation and Data Reduction}

\author{
2.1. Swift
}

The Swift BAT (Gehrels et al. 2004) detected a burst from an unknown source at 09:45:46 UT on 2021 June 3 (trigger number 1053220) and immediately pointed to the source direction (Palmer et al. 2021). The Swift XRT (Burrows et al. 2005) obtained X-ray data in the WT mode for $62 \mathrm{~s}$ from $97 \mathrm{~s}$ after the BAT trigger and then in the PC mode for $\sim 1.7 \mathrm{ks}$ from $1.1 \mathrm{hr}$ after the burst. The XRT observations determined the source position (J2000.0) to be R.A. $=15^{\mathrm{h}} 55^{\mathrm{m}} 08.66$ and decl. $=-54^{\circ} 03^{\prime} 41^{\prime \prime} 1$ with an uncertainty radius of 2 !" 2 at a $90 \%$ confidence level (Evans 2021). We adopt this source position for all analyses in this Letter. The BAT detected another four short bursts from the same direction, as summarized in Appendix Table B1. We analyzed the BAT data using the standard HEASoft BAT pipelines (version 6.28), following the same procedure described in Lien et al. (2016); these results are presented in Section 3.3.

We analyzed the XRT data obtained on June 3, 4, 5, and 7. The observation IDs (ObsIDs) used in this Letter are listed in Appendix Table A1. The observations on June 4, 5, and 7 were carried out in the WT mode for a total of $8.9 \mathrm{ks}$. We processed the data through the standard procedure of FTOOLS xrtpipeline with the default filtering criteria. For PC mode data, we extracted source photons from a circular region with a 20 pixel radius ( 1 pixel $=2$ !" 36) centered at the target, whereas we collected background spectra from a source-free region with a similar (20 pixel) radius located far $\left(>2^{\prime}\right)$ from the source. For the one-dimensional WT mode data, we extracted the source spectra from a rectangular strip of length 40 pixels $\left(\sim 94^{\prime \prime}\right)$. The background was estimated using a source-free region of a similar size located $\sim 2^{\prime}$ from the source position. We used the latest available RMF file in CALDB version 20210504 and generated the ARF files with the xrtmkarf tool.

\subsection{NICER}

NICER (Gendreau et al. 2016), on board the International Space Station, began X-ray observations of the source at 11:21:31 UT on 2021 June 3, $1.6 \mathrm{hr}$ after the first short burst detected with Swift BAT. This initial NICER observation was $2.4 \mathrm{ks}$ long in exposure (ObsID 4202190101), and it was followed by high-cadence monitoring (see Appendix Table A2) carried out almost daily for 29 days under an approved Cycle 3 proposal. Each ObsID had roughly $2 \mathrm{ks}$ exposure and was divided into several continuous good time intervals (GTIs) with exposures of a few hundred seconds for each.

The NICER's X-ray Timing Instrument (XTI) has 52 onorbit active modules, each of which consists of coaligned X-ray concentrators and silicon drift detectors. The XTI has a time resolution of $<100 \mathrm{~ns}$, and the total effective area is about $1,800 \mathrm{~cm}^{2}$ at around $1.5 \mathrm{keV}$. We performed the standard analysis procedures using NICERDAS (version 2020-0423_V007a) in HEASoft 6.27.2 and the NICER calibration database (version 20200722). We generated level 2 cleaned events with the nicerl2 command. For the barycentric correction, we used barycorr with Jet Propulsion Laboratory solar system development ephemeris DE405 for the source 
coordinates stated above (Standish 1998). For timing analyses and burst searches, we utilized all 52 active modules. For spectral studies, we further excluded module numbers 14 and 34 to avoid potential contamination by instrumental noise in the soft energy band. The background spectral model is generated using the 3C50 background model with the nibackgen 3 c50 command (Remillard et al. 2021). ${ }^{33}$

\subsection{NUSTAR}

NuSTAR (Harrison et al. 2013) observed Swift J1555.2 -5402 on June $5-6$ for a $38.4 \mathrm{ks}$ exposure and two additional contemporaneous observations with NICER on June $9(25 \mathrm{ks})$ and $21(29 \mathrm{ks})$. We processed and filtered the NuSTAR data following the standard procedures with HEASoft version 6.28 and CALDB version 20210524 using the nupipline and nuproducts commands. We extracted on-source and background spectra from circular regions of $80^{\prime \prime}$ radius centered at the source position and in a source-free region, respectively. The background-subtracted source count rate of FPMA was about 1 count s$^{-1}$ in the 3-79 keV band. For the spectral fitting, we grouped source spectra using the grppha tool of HEASoft, such that each spectral bin would have a minimum of 50 counts.

\subsection{Deep Space Network}

We carried out radio observations of Swift J1555.2-5402 for a total exposure of roughly $10.8 \mathrm{hr}$ at five epochs during 2021 June 4-12 using different Deep Space Network (DSN) radio telescopes (see Table A4). Simultaneous dual-frequency bands, with center frequencies at 2.2 ( $S$ band) and 8.4 ( $X$ band) $\mathrm{GHz}$, were used for all observations. We used a single circular polarization mode for DSS-34 and DSS-36, whereas a dual circular polarization mode was used at each frequency band for DSS-43.

We recorded the data in filter-bank mode with a time resolution of $512 \mu$ s and frequency resolution of $1 \mathrm{MHz}$ using the pulsar machine in Canberra. The data processing procedure follows similar steps to those presented in earlier studies of pulsars and magnetars with the DSN (e.g., Majid et al. 2017; Pearlman et al. 2018, 2019). After first flattening the bandpass response in each data set, we removed the low-frequency variations in the temporal baseline of each frequency channel by subtracting the moving average from each data point with a time constant of $10 \mathrm{~s}$. The sample times were then corrected to the solar system barycenter.

\subsection{VERA (K Band)}

The $20 \mathrm{~m}$ diameter Ishigaki-jima station of the VLBI Exploration of Radio Astrometry (VERA) conducted a targetof-opportunity observation of this source at an observation frequency of $22 \mathrm{GHz}(1.3 \mathrm{~cm}, K$ band) with a bandwidth of $512 \mathrm{MHz}$. The acquired data for $1 \mathrm{hr}$ at 14:40-15:40 UT on 2021 June 6 were processed and folded to explore radio pulsations both with and without assuming the rotation period. Because the data quality was limited due to a low elevation of the object and bad weather conditions, we could only set an upper limit of the peak flux density of $1.02 \mathrm{Jy}(1 \sigma)$.

\footnotetext{
33 https://heasarc.gsfc.nasa.gov/docs/nicer/tools/nicer_bkg_est_tools.html
}

\section{Analysis and Results}

\subsection{X-Ray Timing Analyses}

Figure 1 shows the time series of the physical parameters of Swift J1555.2-5402 during NICER monitoring for the 29 days from shortly after the onset of the outburst until July 1 . In constructing the time series, we first derived the pulsar spin ephemeris, for which we used a Gaussian pulse template and constructed pulse times of arrival (TOAs) with an integration time of $300 \mathrm{~s}$ and a minimum exposure of $200 \mathrm{~s}$ contained in each bin, using the script photon_toa.py from NICERsoft. ${ }^{34}$ The timing analysis was carried out over $2-8 \mathrm{keV}$, where the energy range was determined from a $Z_{n}^{2}$ search with $n=2$ to optimize the pulse significance (Buccheri et al. 1983). We used the Python-based package for high-precision timing analysis "PINT" (Luo et al. 2021; version 0.8.2) to compute the best timing model through a weighted least-squares fit to the TOAs. The TOAs were found to be well described by a fifthorder polynomial model, as summarized in Table 1, for the spin evolution of Swift J1555.2-5402. The best-fit frequency and its derivatives are $\nu=0.258997103(8) \mathrm{Hz}, \dot{\nu}=-2.04(5) \times$ $10^{-12} \mathrm{~Hz} \mathrm{~s}^{-1}, \ddot{\nu}=-4.50(13) \times 10^{-18} \mathrm{~Hz} \mathrm{~s}^{-2}, \quad \nu^{(3)}=$ $-1.10(10) \times 10^{-23} \mathrm{~Hz} \mathrm{~s}^{-3}, \nu^{(4)}=3.59(15) \times 10^{-29} \mathrm{~Hz} \mathrm{~s}^{-4}$, and $\nu^{(5)}=1.59(14) \times 10^{-34} \mathrm{~Hz} \mathrm{~s}^{-5}$ at barycentric epoch $T_{0}=$ MJD 59,382.7549. NICER's sensitivity enables our measurement up to fifth order in frequency with TOAs of only $300 \mathrm{~s}$.

In Figures 2(a)-(d), we present the energy-resolved and background-subtracted pulse profiles of Swift J1555.2-5402 in the $2-3$ and $3-8 \mathrm{keV}$ bands with NICER and $3-8,8-12$, and $12-20 \mathrm{keV}$ with NuSTAR, where estimates of the background rates were made with the nibackgen3C50 tool for the NICER data and based on the measured rate in the background region for the NuSTAR data. The soft X-ray profile $(2-12 \mathrm{keV})$ shows a single-peaked, nearly sinusoidal shape, while the pulsation in the hard X-ray band ( $\gtrsim 12 \mathrm{keV}$ ) was not detected. We further divided the data into finer energy bands and calculated, in Figure 2(f), the time-averaged rms pulsed fraction (PF) as defined in Bildsten et al. (1997) and Woods et al. (2004). Both the NICER and NuSTAR observations suggest that the rms PF increases from $\sim 30 \%$ in the softer band $(3-4 \mathrm{keV})$ to a maximum of $\sim 40 \%$ at around $7 \mathrm{keV}$ and then decreases with energy to $\lesssim 10 \%$. We also found that the $\mathrm{PF}$ in the $3-8 \mathrm{keV}$ range remained almost constant during the observed period (Figure 1(e)).

\subsection{X-Ray Spectral Analyses}

The right panels of Figure 1 show the long-term spectral properties of Swift J1555.2-5402 obtained with Swift, NICER, and NuSTAR data. Here we applied a single-temperature blackbody multiplied by the Tuebingen-Boulder interstellar absorption model (tbabs*bbodyrad in Xspec terminology) to derive the physical parameters at each epoch (Wilms et al. 2000). The first data point in each of the right panels corresponds to the initial $1.7 \mathrm{ks}$ Swift PC spectrum obtained $\sim 1.1 \mathrm{hr}$ after the BAT trigger, which is well fitted with the model above $\left(\chi^{2}\right.$ of 113 for 128 degrees of freedom, dof). We derived a best-fit hydrogen column density of $N_{\mathrm{H}}=6.8_{-1.7}^{+2.0} \times 10^{22} \mathrm{~cm}^{-2}$ and blackbody temperature of $k T=1.26_{-0.16}^{+0.20} \mathrm{keV}$. The absorbed 2-10 keV flux is $6.8_{-0.8}^{+0.9} \times 10^{-11} \mathrm{erg} \mathrm{s}^{-1} \mathrm{~cm}^{-2}$. This flux is significantly higher than those in the following observations with the Swift/XRT in the WT mode and NICER monitoring.

\footnotetext{
${ }^{34}$ https://github.com/paulray/NICERsoft/
} 

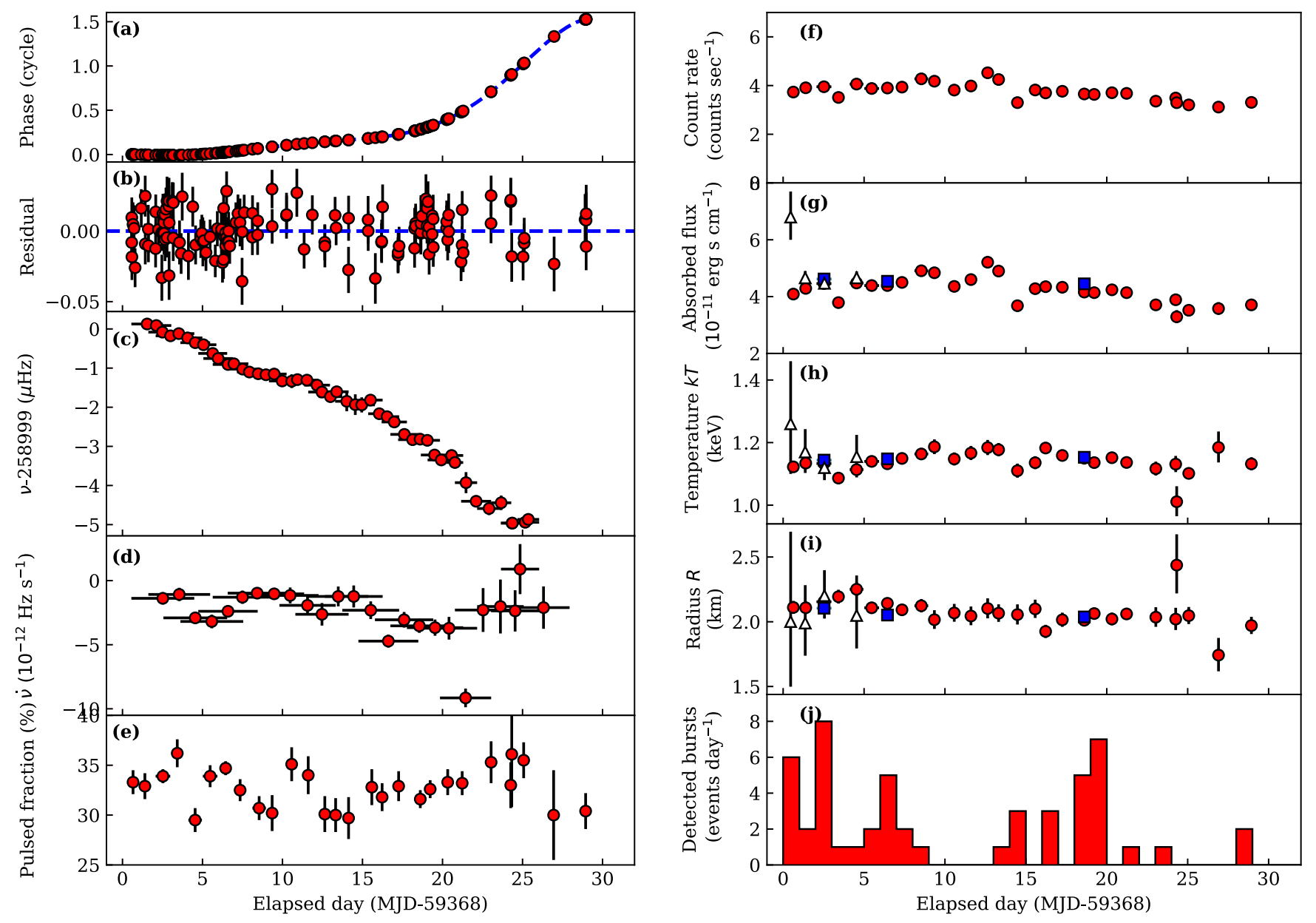

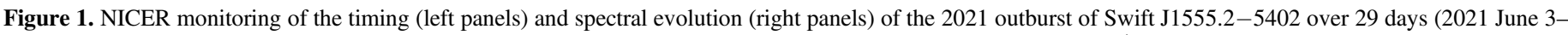

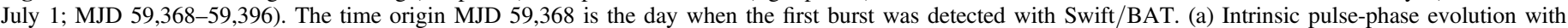

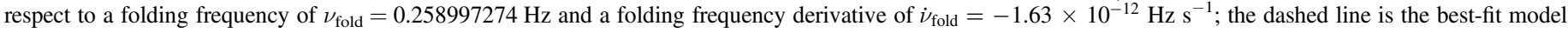

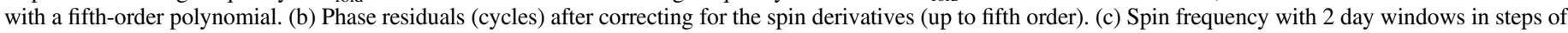

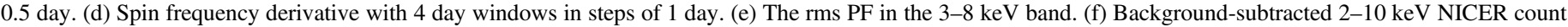

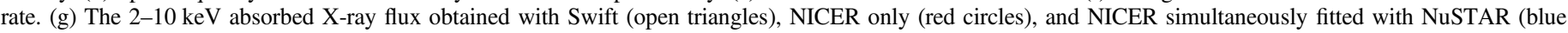

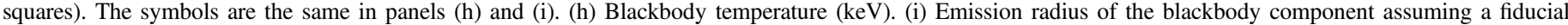
distance of $10 \mathrm{kpc}$. (j) Number of short bursts per day detected with NICER and Swift/BAT. Error bars are at a $68 \%$ confidence limit in these plots.

The subsequent daily NICER spectra $(1.7-10 \mathrm{keV})$ were systematically fitted with the same model with the hydrogen column density tied to be the same value among all NICER spectra at $N_{\mathrm{H}}=(8.88 \pm 0.12) \times 10^{22} \mathrm{~cm}^{-2}$. Each observation has $\sim 4$ counts $\mathrm{s}^{-1}$ (Figure 1(f)). The reduced $\chi^{2}$ values were $\sim 0.9-1.2$ for $\sim 100-400$ dof. No spectral variation during the initial monitoring was found. The absorbed and unabsorbed $2-10 \mathrm{keV}$ fluxes were $\sim 4.3 \times 10^{-11}$ (Figure 1(g)) and $\sim 7.5 \times 10^{-11} \mathrm{erg} \mathrm{s}^{-1} \mathrm{~cm}^{-2}$, respectively. The derived temperature and emission radius were constant at $\sim 1.1 \mathrm{keV}$ (Figure 1(h)) and $\sim 2 d_{10} \mathrm{~km}$ (Figure 1(i)), respectively (Section 3.5). These NICER parameters are consistent with those obtained with the WT mode data of Swift/XRT.

We performed joint spectral fits of three observations of NICER and NuSTAR on June 5-6, 9, and 21. Since the NICER spectra showed no significant time variation, we extracted the NICER spectra for the period on the same day as NUSTAR and regarded them as simultaneous, even if their observation periods were not fully simultaneous. The column densities among the three epochs are tied to the same value. The best-fit spectral model is shown in Figure 3. In addition to the soft X-ray blackbody component, a hard X-ray component above $10 \mathrm{keV}$ was detected with $3 \sigma$ significance extending up to at least $40 \mathrm{keV}$. The hard X-ray flux, when fitted by the power-law model, was (7-9) $\times 10^{-12} \mathrm{erg} \mathrm{s}^{-1} \mathrm{~cm}^{-2}$ in the $10-60 \mathrm{keV}$ band with a power-law photon index of 1.2-1.7. We also performed a combined fit of all three epochs, given that no significant spectral change was observed between them. The resultant average $\nu F_{\nu}$ is shown in the right panel of Figure 3 and Table 1 . The $\nu F_{\nu}$ plot shows that the hard $\mathrm{X}$-ray component above $10 \mathrm{keV}$ exceeds the high-energy tail of the soft blackbody component.

\subsection{Short Burst Analyses}

Swift BAT detected five short bursts, as summarized in Appendix Table B1. For the first detected burst on June 3 (an onboard trigger), we used the data from $T-240$ to $T+100 \mathrm{~s}$, where $T$ is the burst detection time, whereas we used the $\sim 3 \mathrm{~s}$ interval events collected through subthreshold triggers ${ }^{35}$ in our analyses of the other bursts. The BAT event data have a time

\footnotetext{
$\overline{35}$ These are also called failed triggers, which are detections that pass the rate trigger criteria but fail the image detection threshold.
} 
Table 1

Summary of the Timing and Spectral Properties of Swift J1555.2-5402

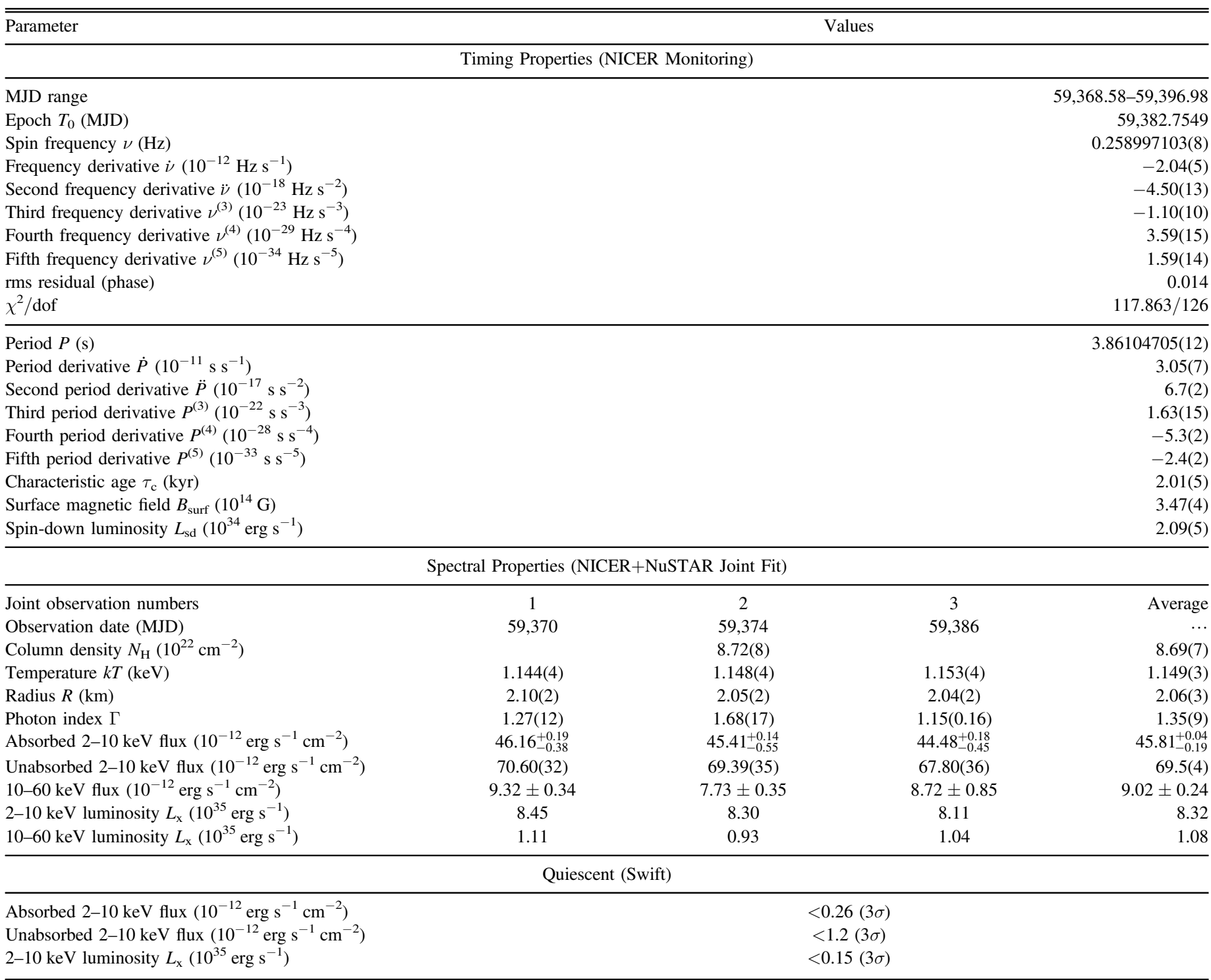

Note. The column density of the three joint NICER and NuSTAR spectral fittings is fixed to the same value. The X-ray luminosity and radius are calculated on an assumption of a fiducial distance of $10 \mathrm{kpc}$. Quoted errors indicate the $68 \%$ confidence limit. The quiescent X-ray upper limits are derived with an assumed temperature of $0.45 \mathrm{keV}$ (see the text).

resolution of $\sim 100 \mu$ s (Barthelmy et al. 2005). The BAT temporal analysis utilizes light curves binned in 1, 2, and $4 \mathrm{~ms}$. Our BAT spectral analysis was performed using spectra created with the $T_{90}$ duration of each burst, which is the duration that covers $90 \%$ of the burst emission. All of the spectra were successfully fitted with a single blackbody model (bbodyrad model in Xspec), except for the one on June 7, the statistics of which were too poor to give meaningful constraints.

We also searched the 2-8 keV NICER event data for short bursts using the Bayesian block technique ${ }^{36}$ (Scargle et al. 2013) and the data filtering criteria in Appendix B. The blocks with high backgrounds and durations longer than $\sim 1 \mathrm{~s}$ are further filtered out on the basis of comparison between the housekeeping data (mkf files), multiple blocks in one burst, and blocks close to the GTI boundaries. We used the Poisson probability to

\footnotetext{
36 https://docs.astropy.org/en/stable/api/astropy.stats.bayesian_blocks.html
}

determine the significance of detecting a number of photons in a block, where the nonburst count rate was calculated from $1 \mathrm{~s}$ intervals close in time to the bursts. We identified 45 short bursts exceeding a $5 \sigma$ detection significance, as summarized in Appendix Table B2. The average duration of the bursts was $23 \pm 17 \mathrm{~ms}$, and 13 photons were detected in a burst, on average. Note that burst $27-1$ on the list occurred during the tail of burst 27, and we included it in burst 27 in the calculation.

We stacked the detected bursts to obtain an average spectrum and found it to be equally well fitted with both a single blackbody (tbabs $*$ bbodyrad) and a power-law (tbabs $*$ pegpwrlw) with Cash statistics of $C$-stat $=261.1$ and 264.0, respectively, with 310 dof. When fixing the absorption column density at $N_{\mathrm{H}}=$ $8.72 \times 10^{22} \mathrm{~cm}^{-2}$ (Table 1), the former model gave a blackbody temperature of $2.9_{-0.5}^{+0.8} \mathrm{keV}$, whereas the latter gave a photon index of $0.0 \pm 0.2$. Using the blackbody model, we find an average unabsorbed flux of $(1.3 \pm 0.1) \times 10^{-8} \mathrm{erg} \mathrm{s}^{-1} \mathrm{~cm}^{-2}$ in the 

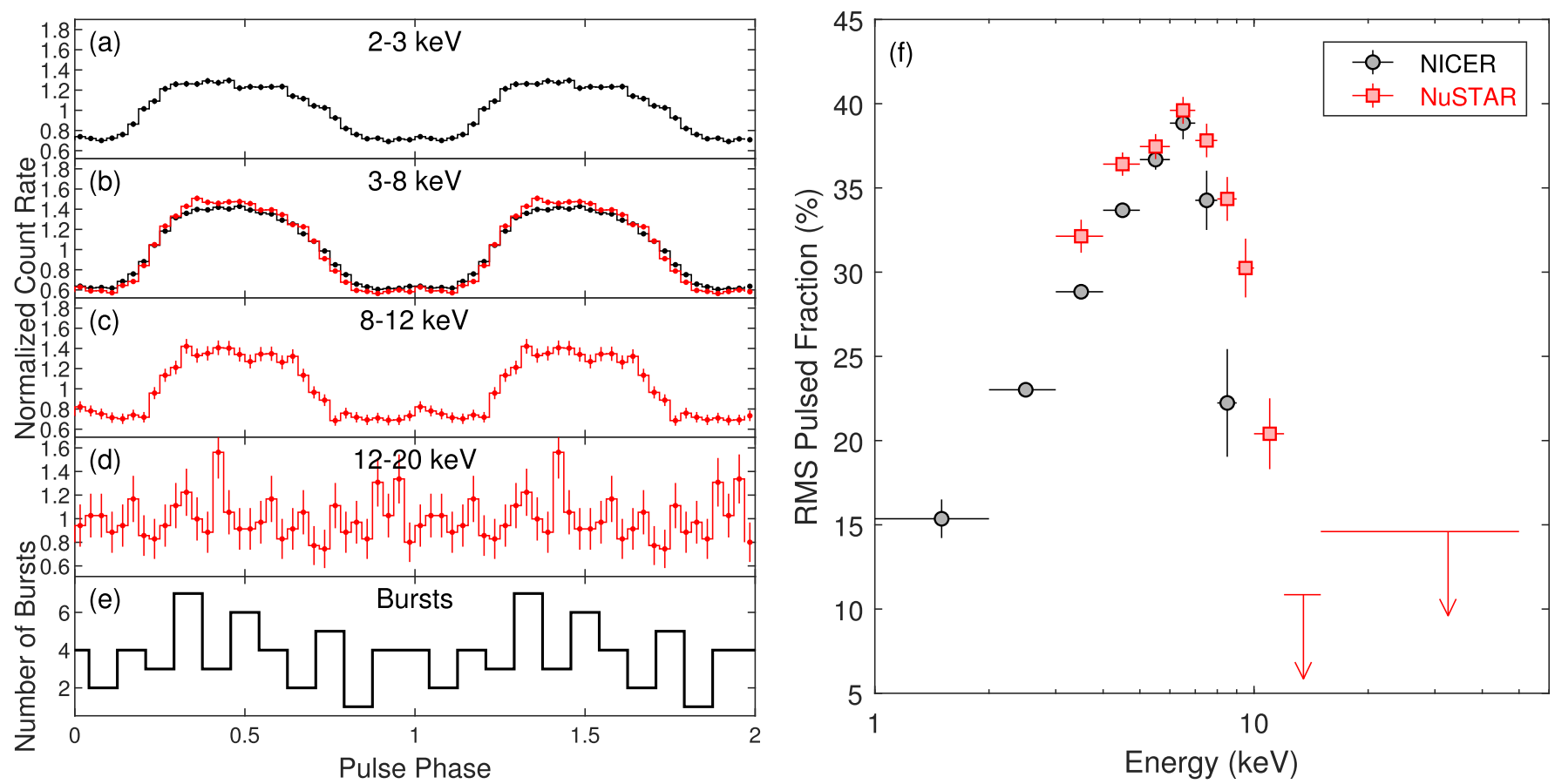

Figure 2. Panels (a)-(d) are background-subtracted X-ray pulse profiles of Swift J1555.2-5402 in the 2-3, 3-8, 8-12, and 12-20 keV bands, respectively, taken with (black) NICER and (red) NuSTAR. The amplitudes are normalized relative to the mean count rate. Two cycles are shown in this figure for clarity. Error bars indicate $1 \sigma$ uncertainties. Panel (e) shows the phase distribution of short bursts. Panel (f): rms PF as a function of energy. The pulsation above $10 \mathrm{keV}$ cannot be significantly seen. We estimate $2 \sigma$ upper limits for the last two energy bins.
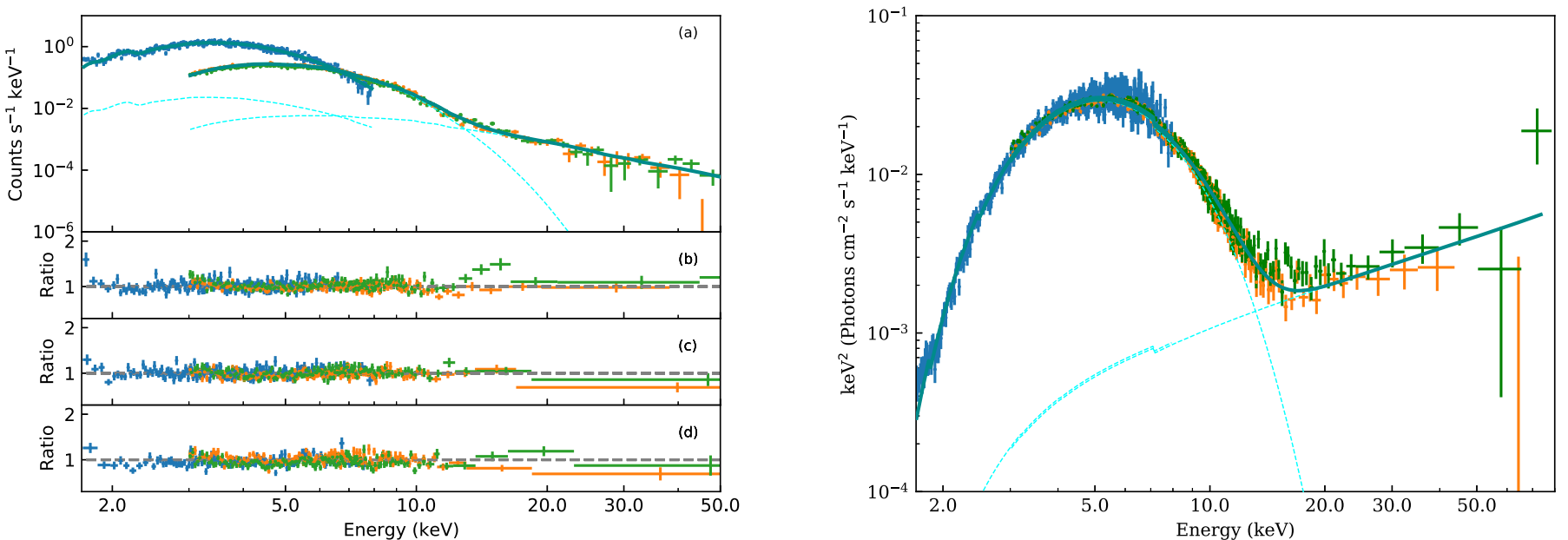

Figure 3. Left: spectral fitting of the joint NICER and NuSTAR data of Swift J1555.2-5402. Panel (a) shows the background-subtracted response-inclusive spectra obtained on June 5 and the best-fit model (dark cyan solid line) with its blackbody and power-law components (cyan dashed lines). Photoelectric absorption is not corrected. Panels (b)-(d) show spectra obtained on June 5,9, and 21, divided by the best-fit model to the first epoch shown in panel (a). Right: best-fit $\nu F_{\nu}$ spectra of NICER and NuSTAR FPMA and FPMB for the three epochs combined. In both panels, NICER and NuSTAR FPMA and FPMB data are shown in blue, orange, and green, respectively.

$2-8 \mathrm{keV}$ range. Assuming a distance of $10 \mathrm{kpc}$, the blackbody radius is estimated to be $7.4 \pm 1.6 \mathrm{~km}$. The fluences of detected bursts are calculated to be in the range of $(1-13) \times 10^{-10} \mathrm{erg} \mathrm{cm}^{-2}$ with an assumed blackbody spectrum of $k T=2.8 \mathrm{keV}$ using the WebPIMMs Appendix (Appendix Table B2). One of the NICER bursts was simultaneously detected with Swift BAT (Appendix Figure B2).

\subsection{DSN Radio Analyses}

We dedispersed the data of each epoch with trial Dispersion Measures (DMs) between zero and $5000 \mathrm{pc} \mathrm{cm}^{-3}$ and subsequently searched each resulting time series for both periodic and single-pulse emission. We found no statistically significant periods with a signal-to-noise ratio $(\mathrm{S} / \mathrm{N})$ above 7.0 after folding individual dedispersed time series modulo period candidates from PRESTO's accelsearch package (Ransom et al. 2002). In addition, we folded the dedispersed time series at each DM trial using the timing model from NICER in Table 1 but found no evidence of radio pulsations at the $S$ or $X$ band during any of our observations. For each epoch, we place $7 \sigma$ upper limits on the magnetar's flux density, assuming a duty cycle of $10 \%$ (see Table A4). Based on our longest observation with DSS-43, the $70 \mathrm{~m}$ radio telescope in Tidbinbilla, Australia, we obtain $7 \sigma$ upper limits on the magnetar's flux of $<0.043 \mathrm{mJy}$ at the $S$ band and $<0.026 \mathrm{mJy}$ at the $X$ band. 
We also searched the dedispersed time series at each frequency band for radio bursts using a matched filtering algorithm, where the time series was convolved with boxcar functions with logarithmically spaced widths between $512 \mu$ s and $150 \mathrm{~ms}$. Candidates with a detection $\mathrm{S} / \mathrm{N}$ above 7.0 were saved and classified using the FETCH software package (Agarwal et al. 2020). The dynamic spectra of the candidates were also visually inspected for verification. We detected no radio bursts during the radio observations and placed $7 \sigma$ upper limits on the fluence of individual bursts during each epoch at both the $S$ and $X$ bands (see Table A4). On 2021 June 5 and 6, we detected two X-ray bursts, with widths of $w=15.91$ (see Table B2; burst 8) and 0.27 ms (see Table B2; burst 10), during overlapping radio and X-ray observations. However, no prompt radio emission (within $\pm 10 \mathrm{~s}$ of the X-ray burst time) was detected above a $7 \sigma$ fluence detection threshold of $1.6 \sqrt{w / 1 \mathrm{~ms}}$ and $0.61 \sqrt{w / 1 \mathrm{~ms}} \mathrm{Jy} \mathrm{ms}$ at the $S$ and $X$ bands, respectively.

\subsection{Search for a Counterpart}

We searched the Swift archival data for a serendipitous detection of Swift J1555.2-5402 in its quiescent state. Two observations in 2012 (ObsIDs 00042728001 and 00042729001) covered the location of this source as part of the Swift XRT Galactic plane survey program (Reynolds et al. 2013). The total exposure is $1055 \mathrm{~s}$. We find no X-ray source at this position within a $30^{\prime \prime}$ radius. The $3 \sigma$ upper limit of the count rate is estimated to be $5.0 \times 10^{-3}$ counts $\mathrm{s}^{-1}$ within this radius. Then, the $3 \sigma$ upper limits of the $2-10 \mathrm{keV}$ absorbed and absorptioncorrected fluxes are calculated to be $5.0 \times 10^{-13}$ and $9.0 \times$ $10^{-13} \mathrm{erg} \mathrm{s}^{-1} \mathrm{~cm}^{-2}$, respectively, on the assumption of an absorbed blackbody spectrum with $N_{\mathrm{H}}=8.72 \times 10^{22} \mathrm{~cm}^{-2}$ and $k T=1.1 \mathrm{keV}$. Yet the magnetar surface temperature in quiescence is considerably smaller than that during outburst epochs, with an average of about $0.45 \mathrm{keV}$ among the population (Olausen \& Kaspi 2014). Hence, we estimate $2-10 \mathrm{keV}$ absorbed and absorption-corrected fluxes of about $2.6 \times 10^{-13}$ and $1.2 \times 10^{-12} \mathrm{erg} \mathrm{s}^{-1} \mathrm{~cm}^{-2}$, respectively. This corresponds to an upper limit of the $2-10 \mathrm{keV}$ quiescent X-ray luminosity of $1.5 \times 10^{34} \mathrm{erg} \mathrm{s}^{-1}$ (bolometric thermal luminosity of $4.8 \times$ $10^{34} \mathrm{erg} \mathrm{s}^{-1}$ ) at $10 \mathrm{kpc}$.

The characteristic age of Swift J1555.2-5402 is inferred to be $2.01 \mathrm{kyr}$ (see Table 1). If we assume that its true age is comparable to its characteristic age, we expect to find a young supernova remnant (SNR) surrounding the neutron star. Detection of an associated SNR, combined with a possible proper-motion measurement, would be helpful and can be important for constraining the magnetar's true age given that the characteristic age may be unreliable due to underlying assumptions about the neutron star rotation period at the birth and braking index. Our search of archival radio, infrared, and X-ray data (e.g., Green 2019) for an SNR or a pulsar-wind nebula coinciding at the position of Swift J1555.2-5402, $(l, b)=(327.872,-0.335)$ in the galactic coordinates using SkyView $^{37}$ fails to yield any convincing candidates.

\section{Discussion and Conclusion}

\subsection{Timing and Spectral Characteristics of the New Magnetar}

High-cadence monitoring with NICER over 1 month allows us to measure the spin ephemeris of the new source

\footnotetext{
37 https://skyview.gsfc.nasa.gov
}

Swift J1555.2-5402 (Table 1). Refined from the initial reports in GCNs and ATels (Coti Zelati et al. 2021b; Ng et al. 2021), the period and its time derivative are measured to be $3.86104705(12) \mathrm{s}$ and $3.05(7) \times 10^{-11} \mathrm{~s} \mathrm{~s}^{-1}$, respectively. The combination of the two values falls within the distribution of known magnetars on the $P-\dot{P}$ diagram (Figure 4(a)). Assuming the standard rotating magnetic dipole model and a braking index of $n=3$, these timing parameters correspond to a characteristic age of $\tau=2.01(5) \mathrm{kyr}$, surface magnetic field strength of $B_{\text {surf }}=3.47(4) \times 10^{14} \mathrm{G}$, and spin-down luminosity of $L_{\mathrm{sd}}=2.09(5) \times 10^{34} \mathrm{erg} \mathrm{s}^{-1}$. This source was classified as a magnetar based on the measured strong magnetic field. The derived characteristic age suggests that it is one of the youngest magnetars among the known ones. The suggestion is supported by the observed strong timing noise, which requires a model with high-order polynomials (Section 3.1), similar to that of the young magnetar Swift J1818.0-1607 (Hu et al. 2020). We caution that the derived pulsar parameters during the outburst may deviate from those in the quiescent state (e.g., Younes et al. 2017a; Archibald et al. 2020). Frequency derivatives are known to fluctuate during magnetar outbursts, with variations of a factor of 1-50 (see, e.g., Dib et al. 2012; Dib \& Kaspi 2014; Levin et al. 2019). Thus, the accuracy of the inferred parameters $B_{\text {surf }} \propto \sqrt{\dot{P}}, \tau \propto \dot{P}^{-1}$, and $L_{\text {sd }} \propto \dot{P}$ relative to the quiescent values still has uncertainties due to this $\dot{P}$ variation.

We detect a hard X-ray tail above $10 \mathrm{keV}$ with NuSTAR, extending up to at least $40 \mathrm{keV}$ with $3 \sigma$ significance. The spectral energy distribution shows that the hard X-ray component is distinguished from the blackbody component, which should originate from the stellar surface (Figure 3). The existence of the distinctive hard tail is further supported by the steep drop in the energy-dependent PF above $10 \mathrm{keV}$ (Figure 2(f)). Two-component spectra of this kind are reported from other persistently bright and transient magnetars (Kuiper et al. 2006; Enoto et al. 2010a; Younes et al. 2017b). The lack of pulsations $(\mathrm{PF}<10 \%)$ in the hard $\mathrm{X}$-rays may suggest that the hard tail originates in magnetospheric emission that does not have much anisotropy and higher emission altitude than emission from the stellar surface. This may imply a low magnetic impact parameter (e.g., Wadiasingh et al. 2018) for the observer across the pulse for resonant Compton scattering. The $15-60 \mathrm{keV}$ flux of Swift J1555.2-5402, $F_{15-60}=6.21 \pm$ $0.23 \times 10^{-12} \mathrm{erg} \mathrm{s}^{-1} \mathrm{~cm}^{-2}$, is lower than the absorbed $1-10 \mathrm{keV}$ flux $F_{1-10}=4.65_{-0.27}^{+0.19} \times 10^{-11} \mathrm{erg} \mathrm{s}^{-1} \mathrm{~cm}^{-2}$. Accordingly, the broadband hardness ratio of the magnetospheric to surface thermal emissions is $\eta=F_{15-60} / F_{1-10}=0.13$. The hardness ratio $\eta$ of known magnetars $(\eta=0.1-4)$ is suggested to be correlated with the surface magnetic field. Figure 4(d) plots the $\eta$ values of known sources and Swift J1555.2-5402. It shows that the $\eta$ of Swift J1555.2-5402 is not largely off, though apparently smaller than, the proposed correlation (see Equation (3) of Enoto et al. 2017). We note that the measured $\eta$ has some systematic uncertainty. If the yet-unknown long-term frequency derivative of Swift J1555.2-5402 is lower than the value measured during the current outburst, the magnetic field strength in the quiescent state is weaker than our estimate, which places the $\eta$ of Swift J1555.2-5402 closer to the known correlation.

\subsection{X-Ray Luminosity}

Figure 4(b) compares pulsar X-ray luminosity $L_{\mathrm{x}}$ with spindown luminosity $L_{\mathrm{sd}}$. Immediately after the outburst, the 

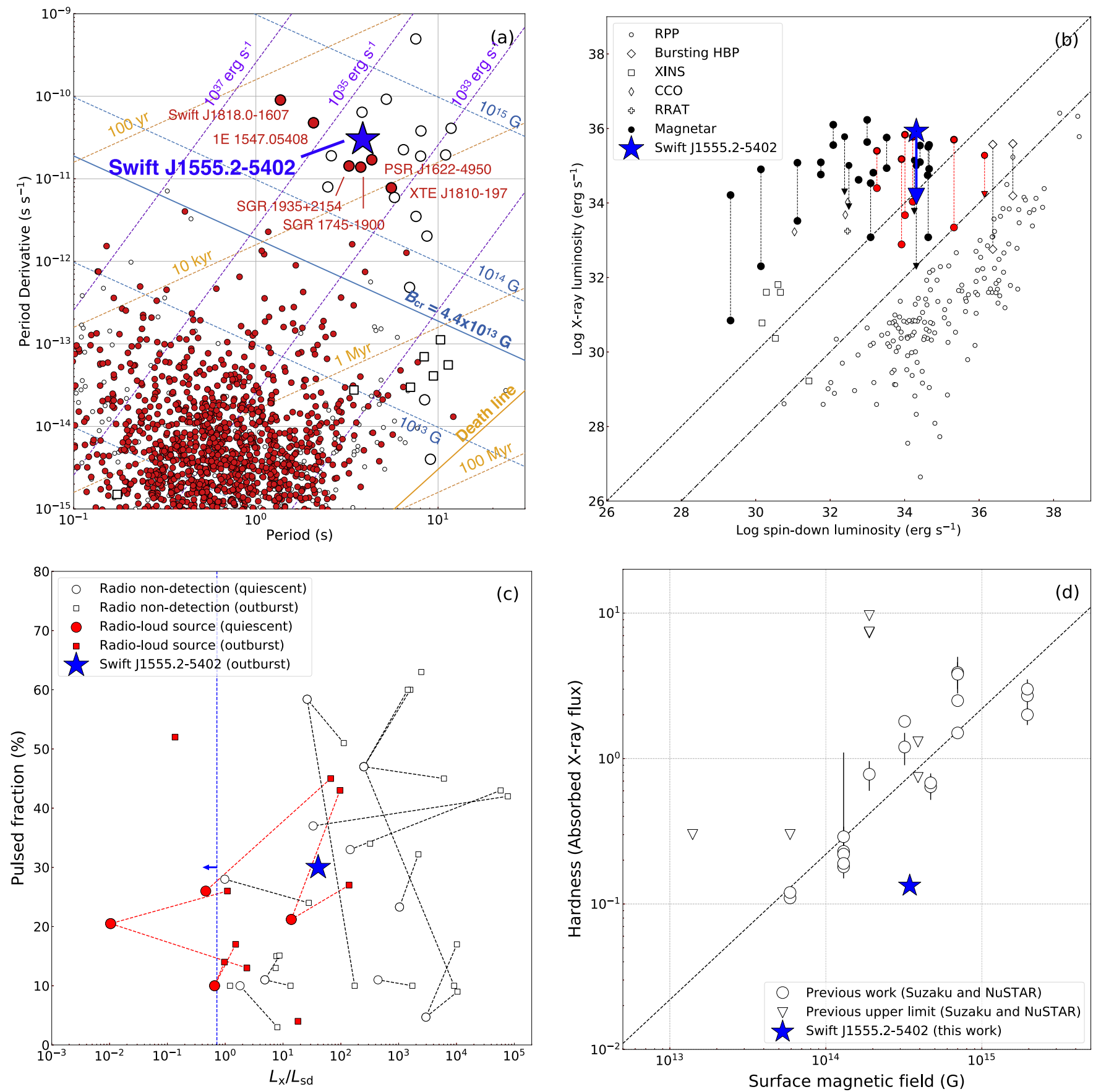

Figure 4. (a) Position of the new magnetar Swift J1555.2-5402 (star) on the $P-\dot{P}$ diagram. Large and small circles indicate magnetars (from the McGill catalog; Olausen \& Kaspi 2014) and canonical rotation-powered pulsars (from the ATNF catalog; Manchester et al. 2005), respectively. Filled red symbols indicate radioemitting pulsars. The lines show constant surface magnetic field strengths, characteristic ages, and spin-down luminosities. Squares in panels (a) and (b) are X-ray-dim isolated neutron stars. (b) Observed X-ray luminosity in the soft X-ray band (including the unpulsed component) compared with the spin-down power for various types of pulsars. The peak X-ray luminosity and quiescent values of magnetars are connected via dashed lines. Red symbols indicate radio-loud magnetars. The two diagonal lines indicate where the X-ray luminosity becomes equal to $100 \%$ and $1 \%$ of the spin-down power. The values and references used in panels (b)-(d) are summarized in Appendix Tables C1 and C2 for magnetars and Enoto et al. (2019) for other pulsars. (c) The PFs as a function of the X-ray luminosity normalized by the spin-down power. Filled red symbols indicate radio-emitting magnetars. Circles and squares represent data in quiescence and during X-ray outbursts, respectively. A dashed line connects observations for the same source. The vertical dashed line with the arrow indicates the region of the quiescent state of Swift J1555.2-5402. (d) Broadband hardness ratio of absorbed X-ray fluxes between 1-10 and 15-60 keV with the best-fit correlation (Enoto et al. 2017).

surface emission $(2-10 \mathrm{keV})$ and total $(2-60 \mathrm{keV})$ luminosity of Swift J1555.2-5402 were $L_{\mathrm{x}}=8.5 \times 10^{35} d_{10}^{2}$ and $9.6 \times$ $10^{35} d_{10}^{2} \mathrm{erg} \mathrm{s}^{-1}$, respectively. At the assumed distance of $10 \mathrm{kpc}$, these are, respectively, 40 and 46 times larger than its spin-down luminosity $L_{\mathrm{sd}}=2.1 \times 10^{34} \mathrm{erg} \mathrm{s}^{-1}$. The observed X-ray luminosity is similar to those of past reported outbursts of transient magnetars. The upper limit on the quiescent $\mathrm{X}$-ray luminosity of Swift J1555.2-5402 is $L_{\text {quie }} \approx 1.5 \times 10^{34} d_{10}^{2}$, corresponding to $L_{\text {quie }} / L_{\text {sd }} \lesssim 0.71 d_{10}^{2}$, which is still up to $2-3$ orders of magnitude higher than those of the rotation-powered pulsars (see Figure 4(b) and Figure 12 of Enoto et al. 2019). The upper limit on the quiescent 
bolometric thermal luminosity $\approx 4.8 \times 10^{34} d_{10}^{2} \mathrm{erg} \mathrm{s}^{-1}$ also makes Swift J1555.2-5402 one of the coolest young magnetars (see, e.g., Potekhin et al. 2015) and is compatible with the $L \sim 2 \times 10^{33} \mathrm{erg} \mathrm{s}^{-1}$ of PSR J1119-6127 (Gonzalez et al. 2005; $\mathrm{Ng}$ et al. 2012; Blumer et al. 2021). The upper limit on the quiescent bolometric thermal luminosity $\approx 4.8 \times 10^{34} d_{10}^{2}$ $\mathrm{erg} \mathrm{s}^{-1} \mathrm{erg} \mathrm{s}^{-1}$ is compatible with $L \sim 2 \times 10^{33} \mathrm{erg} \mathrm{s}^{-1}$ of PSR J1119-6127 (Gonzalez et al. 2005; Ng et al. 2012; Blumer et al. 2021) and could mean that Swift J1555.2-5402 is one of the cooler young magnetars (see, e.g., Potekhin et al. 2015).

\subsection{Peculiarities of the Slow Decline Outburst}

The persistent X-ray flux of most transient magnetars remains in a bright plateau state for a few weeks immediately after the onset of outbursts and then starts to fade over the next several months (Coti Zelati et al. 2018). Typically, the plateau duration and decaying slope are $\tau_{0}=11-43$ days and $p \sim 0.7-2$ (Enoto et al. 2017), respectively, when the X-ray flux $F_{\mathrm{x}}$ is fitted with an empirical formula $F_{\mathrm{x}}(t)=F_{0} /\left(1+t / \tau_{0}\right)^{p}$, where $t$ and $F_{0}$ are the elapsed time and plateau flux, respectively. A peculiarity of Swift J1555.2-5402 is its longlasting outburst. The absorbed X-ray flux stayed nearly stable at around $4 \times 10^{-11} \mathrm{erg} \mathrm{s}^{-1} \mathrm{~cm}^{-2}$ with only a slow decline over a month. There was no apparent rollover of the flux trend as of July 1 . Such long-lasting outbursts are rare for magnetars, with only a few exceptions ever recorded, e.g., the radio-loud magnetar 1E 1547.0-5408.

Another peculiarity of the Swift J1555.2-5402 outburst is the higher temperature $(k T \sim 1.1 \mathrm{keV})$ of the blackbody component than the typical value of known magnetars, $k T \sim 0.3-0.7 \mathrm{keV}$ (Enoto et al. 2017), despite the surface emission radius $R \sim 2 d_{10} \mathrm{~km}$ of Swift J1555.2-5402 being well within the typical range for a neutron star. During the 1 month observation, the slow flux decline originated from the decreasing emission radius rather than the temperature decline. This fact suggests a situation where the hot spot on the neutron star surface responsible for the X-ray emission was shrinking, which is consistent with the twisted magnetosphere model (Kaspi \& Beloborodov 2017).

We detected 45 and 5 short bursts with NICER and Swift/ BAT, respectively, during the initial 2 weeks since discovery of Swift J1555.2-5402 (Figure 1(j)). The burst-active periods of persistently bright magnetars (e.g., SGR 1806-20) are known to be longer ( $\gtrsim 100$ days) than those ( $\lesssim 10$ days) of low-burst rate transient ones (e.g., SGR 0501+4516; Göğüş 2014). The burst-active period of Swift J1555.2-5402 is close to the latter case. We conjecture that repeated bursts as observed in Swift J1555.2-5402 would provide impulsive heating of the surface to sustain the long-lasting decay. To investigate the potential relationship between the bursts and persistent emission on the pulse profile, we plot in Figure 2(e) the phase distribution of the observed bursts. An Anderson-Darling (AD) test suggests that the burst phase distribution differs from a uniform distribution with an AD statistic of 0.42 and corresponding $p$-value of 0.82 . Thus, there is neither a statistical difference between the phase distribution of the bursts and uniform distribution nor a statistically significant correlation between the burst occurrence and the pulse profile so far.

\subsection{Comparison with Radio-emitting Magnetars}

We did not detect any radio emission from this source. It has been long established theoretically that as far as ordinary pulsars are concerned, the occurrence of rotation-powered polar-cap radio emission requires a sufficiently large potential drop $\Delta \Phi$ to generate electron-positron pairs near polar caps (Goldreich \& Julian 1969; Sturrock 1971; Ruderman \& Sutherland 1975; Arons \& Scharlemann 1979); note that the conventional definition of $\Delta \Phi \propto L_{\mathrm{sd}}^{1 / 2}$ implies that radio emission is equivalently related to $L_{\mathrm{sd}}$ (Ho 2013). The observed radio luminosity of rotation-powered pulsars indeed follows the relation (e.g., Arzoumanian et al. 2002). In the absence of magnetically induced nonpotential fields, magnetars that satisfy this condition at their polar caps before crossing the death line should, in principle, be capable of producing coherent radio emission. However, as shown in Figures 4(a)-(c) and summarized in Appendix Table $\mathrm{C} 1$, pulsed radio emission has only been reported from six radio-loud magnetars and a high-B pulsar that exhibited a magnetar-like outburst. It is an open question under what conditions a magnetar becomes radio-loud. The new magnetar Swift J1555.2-5402 is located in the $P-\dot{P}$ parameter space close to radio-loud magnetars (Figure 4(a)). The DSN upper limits on the radio luminosity $\left(\nu L_{\nu}=1.1 \times 10^{28} d_{10}^{2} \mathrm{erg} \mathrm{s}^{-1}\right.$ for the $S$ band and $2.6 \times 10^{28} d_{10}^{2}$ $\mathrm{erg} \mathrm{s}^{-1}$ for the $X$ band) would be much lower than those of the known radio-loud magnetars, $\nu L_{\nu} \sim(0.08-1) \times 10^{30}$ and $\sim(0.01-3) \times 10^{31} \mathrm{erg} \mathrm{s}^{-1}$ at the $S$ and $X$ bands, respectively (Camilo et al. 2007a, 2007b, 2008; Levin et al. 2010, 2012; Shannon \& Johnston 2013; Pennucci et al. 2015; Huang et al. 2021). Therefore, the lack of radio emission suggests the existence of some other physical factors than simply $P$ and $\dot{P}$ that govern radio emission.

One crucial factor is the geometry among the pulsar rotation axis, magnetic axis, line of sight to the observer, and width of any putative radio beam. For example, an anisotropic radio beam aligned to the magnetic axis must cross the line of sight to be detected, and longer-period rotation-powered pulsars tend to have narrower beams (e.g., Lyne \& Manchester 1988; Rankin 1993). The radio nondetection of Swift J1555.2-5402 might suggest that this magnetar may not have a favorable geometry for detection (e.g., Lazarus et al. 2012). Some radioloud magnetars have observational signatures that suggest aligned rotators (i.e., the angle between the magnetic axis and rotation axis is $\lesssim 30^{\circ}$; Camilo et al. 2008; Levin et al. 2012; Lower et al. 2020). As shown in Figure 4(c), the PF both in quiescence and during X-ray outbursts of radio-loud magnetars is lower than that of the other magnetars. A low PF could be due to radio-loud magnetars being observed as near-aligned rotators. The same interpretation can be inferred from the systematic Fourier-decomposition study of X-ray profiles of magnetars in quiescence conducted by Hu et al. (2019); radioloud magnetars have a more sinusoidal pulse profile with a pronounced first Fourier component accompanied by a weaker second component. Another factor for the radio emission is the effects of higher-order (and possibly not curl-free) magnetic field components other than the dipolar field inferred from $P-\dot{P}$, which has already been taken into account. Quantum electrodynamic effects affect the conditions for pair cascades and radio emission when a magnetic field is sufficiently strong, above $10^{13} \mathrm{G}$. For example, if photon splitting occurs in a region above the critical magnetic field, the way the electron/ positron cascade occurs is modified and perhaps quenched, and 
radio pulsation may be suppressed (e.g., Baring \& Harding 2001). Finally, magnetar magnetospheres are considered to be dynamic, and the radio flux, pulse profile, and polarization swing pattern can change significantly within a few days (e.g., Lower et al. 2021). Future monitoring of this new magnetar, Swift J1555.2 -5402 , will be important for understanding the conditions for the magnetar radio emission.

The authors are grateful to the NICER, NuSTAR, Swift, and DSN scheduling and operation teams. We thank Francesco Coti Zelati, Alice Borghese, Nanda Rea, Gian Luca Israel, and Paolo Esposito for helpful discussion about the initial observation and for preparing the GCN reports. We also acknowledge Alice Borghese for her request of the Swift observation at the earliest phase of the outburst. We thank Mareki Honma, Takaaki Jike, Aya Yamauchi, Toshio Terasawa, and Tomoya Hirota for useful comments on the VERA observation and all the staff of Mizusawa VLBI Observatory of NAOJ for operating the VERA array. T.E. and S.K. are supported by JSPS/MEXT KAKENHI grant Nos. 17K18776, 18H04584, 18H01246, 19K14712, and 21H01078. A.B.P. is a McGill Space Institute (MSI) Fellow and a Fonds de Recherche du Quebec-Nature et Technologies (FRQNT) postdoctoral fellow. W.C.G.H. acknowledges support through grant 80NSSC20K0278 from
NASA. NICER research at NRL is supported by NASA. W.A. $M$. is grateful to the DSN scheduling team and the Canberra Deep Space Communication Complex (CDSCC) staff for scheduling and carrying out the radio observations with the DSN. A portion of this research was performed at the Jet Propulsion Laboratory, California Institute of Technology, under a Research and Technology Development Grant through a contract with the National Aeronautics and Space Administration. U.S. government sponsorship is acknowledged.

Facilities: NICER, NuSTAR, Swift, DSN, VERA.

Software: HEAsoft (v6.27.2; NASA High Energy Astrophysics Science Archive Research Center (HEASARC), 2014), astropy (Astropy Collaboration et al. 2013; Price-Whelan et al. 2018), PRESTO (Ransom et al. 2002), FETCH (Agarwal et al. 2020), PINT (v0.8.2; Luo et al. 2021), XSPEC (Arnaud 1996), NICERDAS (v2020-04-23_V007a).

\section{Appendix A List of Observations}

Tables A1-A4 summarize the observations of Swift, NICER, NuSTAR, and DSN, respectively, conducted for this campaign as of July 1.

Table A1

A List of Swift ObsIDs of Swift J1555.2-5402

\begin{tabular}{|c|c|c|c|c|c|c|c|c|}
\hline No. & ObsID & Mode & $\begin{array}{l}\text { Start Time } \\
\text { (UTC) }\end{array}$ & $\begin{array}{l}\text { End Time } \\
\text { (UTC) }\end{array}$ & MJD & $\begin{array}{l}\text { Elapsed } \\
\text { Days }\end{array}$ & $\begin{array}{l}\text { Exposure } \\
\text { (s) }\end{array}$ & $\begin{array}{l}\text { Rate } \\
\text { (cps) }\end{array}$ \\
\hline 1 & 01053220000 & $\mathrm{PC}$ & 2021-06-03Т09:46:24 & 2021-06-03T11:21:33 & $59,368.463$ & 0.06 & 1706 & $0.69 \pm 0.06$ \\
\hline 2 & 00014352001 & WT & 2021-06-04T7:50:02 & 2021-06-04T9:37:00 & $59,369.364$ & 1.0 & 1965 & $0.52 \pm 0.02$ \\
\hline 3 & 00014352002 & WT & 2021-06-05T10:40:43 & 2021-06-05T15:46:23 & $59,370.551$ & 2.1 & 4895 & $0.51 \pm 0.02$ \\
\hline 4 & 00014352003 & WT & 2021-06-07T12:06:00 & 2021-06-07T13:49:00 & $59,372.540$ & 4.1 & 1940 & $0.53 \pm 0.02$ \\
\hline
\end{tabular}

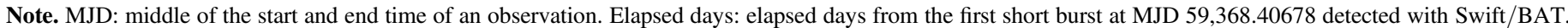
Rate: background-subtracted $2-10 \mathrm{keV}$ count rate of Swift. 
Table A2

A List of NICER ObsIDs of Swift J1555.2-5402

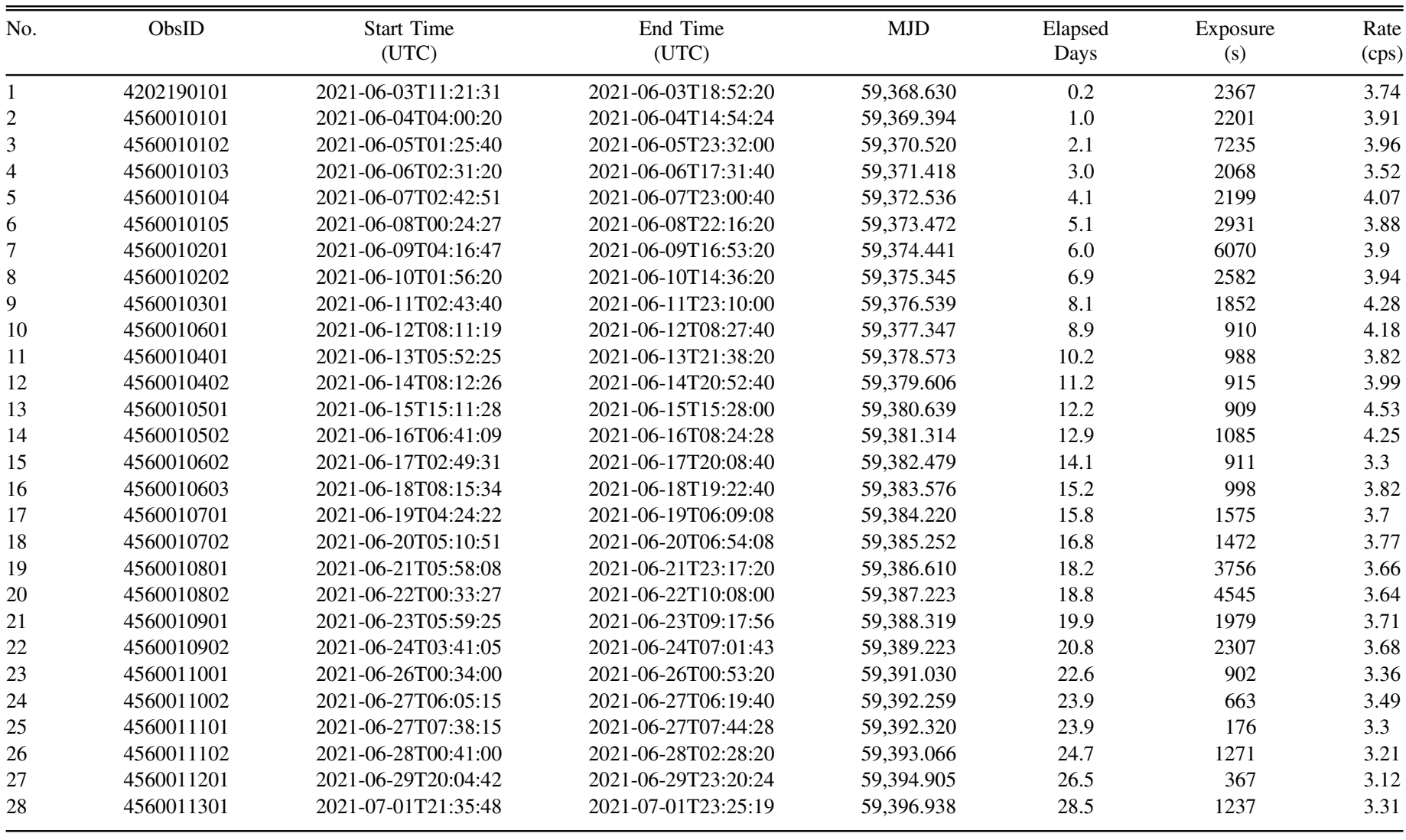

Note. MJD: middle of the start and end time of an observation. Elapsed days: elapsed days from the first short burst at MJD 59,368.40678 detected with Swift/BAT. Rate: background-subtracted $2-10 \mathrm{keV}$ count rate of NICER.

Table A3

A List of NuSTAR ObsIDs of Swift J1555.2-5402

\begin{tabular}{|c|c|c|c|c|c|c|c|}
\hline No. & ObsID & $\begin{array}{l}\text { Start Time } \\
\text { (UTC) }\end{array}$ & $\begin{array}{l}\text { End Time } \\
\text { (UTC) }\end{array}$ & MJD Start & $\begin{array}{l}\text { Exposure } \\
(\mathrm{ks})\end{array}$ & $\begin{array}{c}\text { Rate A } \\
(\mathrm{cps})\end{array}$ & $\begin{array}{c}\text { Rate B } \\
\text { (cps) }\end{array}$ \\
\hline 1 & 90701319002 & 2021-06-05T10:20:48 & 2021-06-06Т06:33:07 & $59,370.43111111$ & 38.4 & 1.12 & 1.03 \\
\hline 2 & 80702313002 & 2021-06-09Т05:39:00 & 2021-06-09T18:00:00 & $59,374.23541667$ & 25.0 & 1.09 & 1.025 \\
\hline 3 & 80702313004 & 2021-06-21T14:05:11 & 2021-06-22Т04:15:00 & $59,386.58693287$ & 28.9 & 0.99 & 0.94 \\
\hline
\end{tabular}

Note. Rates A and B are the 3-79 keV count rate of NuSTAR FPMA and FPMB, respectively.

Table A4

A List of Radio Observations of Swift J1555.2-5402 with the DSN

\begin{tabular}{|c|c|c|c|c|c|c|c|}
\hline No. & Instrument & $\begin{array}{l}\text { Observation } \\
\text { Start Time } \\
\text { (UTC) }\end{array}$ & $\begin{array}{l}\text { Observation } \\
\text { Start Time } \\
\text { (MJD) }\end{array}$ & $\begin{array}{l}\text { Duration } \\
\text { (hr) }\end{array}$ & $\begin{array}{c}\text { Observing } \\
\text { Frequency Band }\end{array}$ & $\begin{array}{c}\text { Mean Flux Density } \\
\left(S_{\text {mean }}^{S \text {-band }} / S_{\text {mean }}^{X \text {-band }}\right) \\
(\mathrm{mJy} / \mathrm{mJy})\end{array}$ & $\begin{array}{c}\text { Radio Burst Fluence }{ }^{\mathrm{c}} \\
\left(\mathcal{F}^{S \text {-band }} / \mathcal{F}^{X \text {-band }}\right) \\
(\text { Jy ms } / \text { Jy ms })\end{array}$ \\
\hline 1 & DSN (DSS-36) & 2021 Jun 4 06:59:02 & $59,369.29099$ & 1.3 & $S$ band $/ X$ band & $<0.27 /<0.10$ & $<1.7 /<0.7$ \\
\hline 2 & DSN (DSS-36) & 2021 Jun 5 11:46:10 & $59,370.49039$ & 2.4 & $S$ band $/ X$ band & $<0.18 /<0.07$ & $<1.6 /<0.61$ \\
\hline 3 & DSN (DSS-43) & 2021 Jun 6 04:49:06 & $59,371.20076$ & 2.2 & $S$ band $/ X$ band & $<0.042 /<0.025$ & $<0.35 /<0.21$ \\
\hline 4 & DSN (DSS-34) & 2021 Jun 10 11:31:12 & $59,375.48000$ & 2.4 & $S$ band $/ X$ band & $<0.20 /<0.11$ & $<1.7 /<0.9$ \\
\hline 5 & DSN (DSS-43) & 2021 Jun 12 04:08:52 & $59,377.17282$ & 2.5 & $S$ band $/ X$ band & $<0.043 /<0.026$ & $<0.39 /<0.24$ \\
\hline
\end{tabular}

Notes. The first two observations (2021 June 4 and 5) and the fourth observation (2021 June 10) were carried out using DSS-36 and DSS-34, two $34 \mathrm{~m}$ diameter radio telescopes in Canberra, Australia, whereas the remaining observations (2021 June 6, and 12) were carried out using DSS-43, the 70 m diameter dish in Canberra. ${ }^{\text {a }}$ The center frequencies at the $S / X$ bands are $2.2 / 8.4 \mathrm{GHz}$, respectively.

$\mathrm{b}$ The $7 \sigma$ upper limits on the mean flux density in each radio frequency band, assuming a $10 \%$ duty cycle. The uncertainties on the mean flux density upper limits are estimated at $15 \%$, primarily due to the uncertainty in the system temperature.

${ }^{\mathrm{c}}$ The $7 \sigma$ upper limits on the radio burst fluence in each radio frequency band, assuming a burst width of $1 \mathrm{~ms}$. The uncertainties on the fluence detection thresholds are estimated at $15 \%$, primarily due to the uncertainty in the system temperature. 


\section{Appendix B \\ Burst Analyses}

Tables B1 and B2 summarize the detected magnetar short bursts by Swift/BAT and NICER, respectively. For the burst search, we recalibrate the event files using nicerl2 with criteria of elv=30 br_earth $=30$ underonly_range $=0$ 300 overonly_range $=0-2$ overonly_expr=' ' $2.0{ }^{\prime \prime}$ ' to maximize the time coverage. The corresponding integrated fluence distribution detected with NICER is shown in Figure B1. It shows a power law-like distribution with a low-fluence

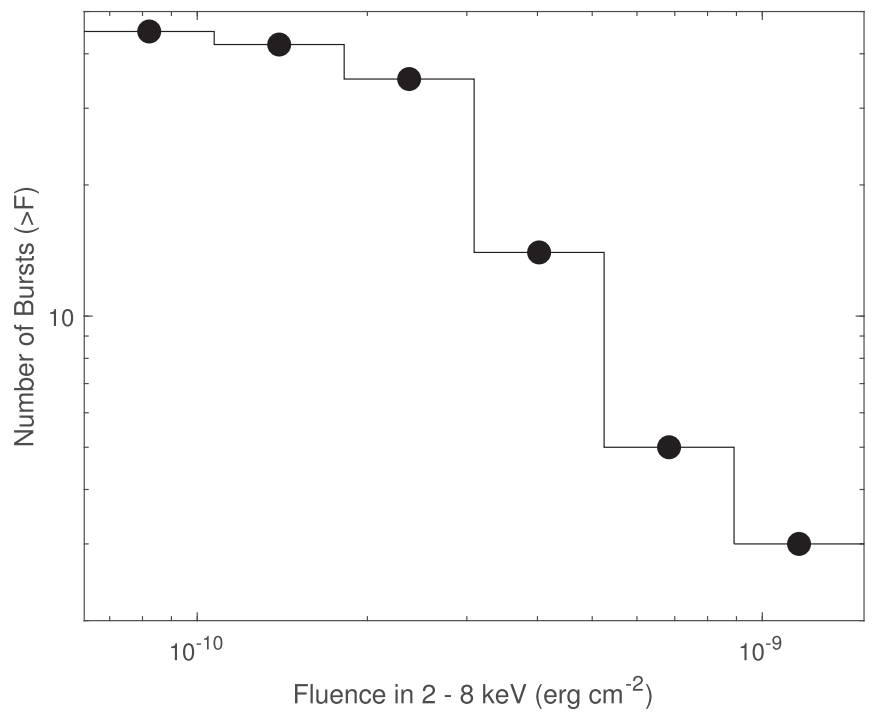

Figure B1. Integrated fluence distribution in $2-8 \mathrm{keV}$ of the detected short bursts from Swift J1555.2-5402 obtained with NICER.
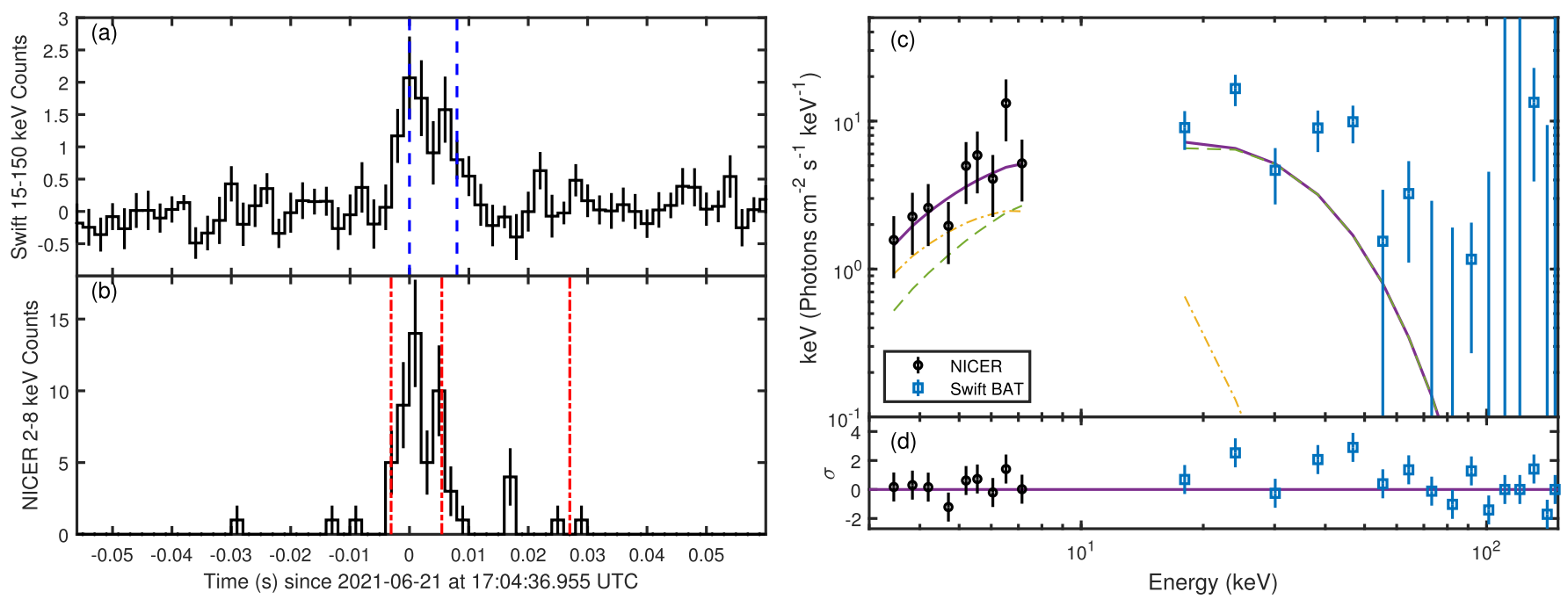

Figure B2. Light curves of a burst detected simultaneously with (a) Swift BAT in 15-150 keV, burst number 5 in Table B1, and (b) NICER in 2-8 keV, numbers 32 and 33 in Table B2. The bin size of the light curves is $2 \mathrm{~ms}$. Blue dashed lines denote the start and end of $T_{90}$ of the Swift light curve, where the red dashed-dotted lines are the boundaries of Bayesian blocks detected with NICER events. The broadband X-ray spectrum of this burst is shown in panel (c), with the best-fit model (purple solid line) consisting of two blackbodies (orange dashed-dotted line and green dashed line). The residuals are shown in panel (d).

Table B1

A List of Short Bursts from Swift J1555.2-5402 Detected with Swift/BAT

\begin{tabular}{|c|c|c|c|c|c|c|c|}
\hline No. & Trigger ID & $\begin{array}{l}\text { Time } \\
\text { (UTC) }\end{array}$ & $\begin{array}{l}\text { Duration } \\
T_{90}(\mathrm{~ms})\end{array}$ & $\mathrm{S} / \mathrm{N}$ & $\begin{array}{c}k T \\
(\mathrm{keV})\end{array}$ & Fluence & $\chi^{2}$ \\
\hline 1 & 1053220 & 2021-06-03Т09:45:46.589 & $12 \pm 2.8$ & 9.9 & $6.66 \pm 0.98$ & $9.09 \pm 2.32$ & 33.98 \\
\hline 2 & 1053653 & 2021-06-05T23:52:04.582 & $14 \pm 4.5$ & 7.3 & $8.53 \pm 1.40$ & $7.47 \pm 2.62$ & 28.54 \\
\hline 3 & 1053961 & 2021-06-07T12:33:40.020 & $4 \pm 2.2$ & 5.0 & $\mathrm{~N} / \mathrm{A}^{\mathrm{a}}$ & $\mathrm{N} / \mathrm{A}^{\mathrm{a}}$ & $\mathrm{N} / \mathrm{A}^{\mathrm{a}}$ \\
\hline 4 & 1056025 & 2021-06-16T14:44:30.489 & $7 \pm 2.8$ & 6.9 & $6.58 \pm 2.22$ & $<3.08$ & 31.20 \\
\hline 5 & 1057131 & 2021-06-21T17:04:36.839 & $12 \pm 4.5$ & 6.9 & $6.47 \pm 2.03$ & $<3.82$ & 55.54 \\
\hline
\end{tabular}

Notes. Reported errors are $90 \%$ confidence for each parameter. Time: burst detection time (UTC) determined as the start time of $T_{90} . \mathrm{S} / \mathrm{N}$ : Signal-to-noise ratio of the BAT image in $15-350 \mathrm{keV} . k T$ : blackbody temperature $(\mathrm{keV})$ when fitted by the single blackbody model. Fluence: burst fluence in the $15-150 \mathrm{keV}$ band $\left(10^{-9} \mathrm{erg} \mathrm{cm}^{-2}\right) \cdot \chi^{2}$ : fitting $\chi^{2}$ values for $57 \mathrm{dof}$.

${ }^{a}$ Burst 3 is too weak to constrain the spectral-fit parameters. 
Table B2

A List of Short Bursts from Swift J1555.2-5402 Detected with NICER

\begin{tabular}{|c|c|c|c|c|c|c|}
\hline No. & ObsID & $\begin{array}{l}\text { Time } \\
\text { (UTC) }\end{array}$ & $\begin{array}{l}\text { Duration } \\
\quad(\mathrm{ms})\end{array}$ & $\begin{array}{c}\text { Significance } \\
\sigma\end{array}$ & Phase & Fluence \\
\hline 1 & 4202190101 & 2021-06-03T13:51:09.341 & 14.94 & 8.80 & 0.085 & $2.6 \pm 0.8$ \\
\hline 2 & 4202190101 & 2021-06-03T13:51:27.181 & 59.18 & 6.38 & 0.711 & $2.4 \pm 0.8$ \\
\hline 3 & 4202190101 & 2021-06-03T13:54:40.275 & 19.79 & 15.47 & 0.717 & $6.3 \pm 1.3$ \\
\hline 4 & 4202190101 & 2021-06-03T14:01:50.242 & 12.95 & 6.75 & 0.076 & $1.9 \pm 0.7$ \\
\hline 5 & 4202190101 & 2021-06-03T18:49:06.251 & 35.90 & 6.35 & 0.166 & $1.9 \pm 0.7$ \\
\hline 6 & 4560010101 & 2021-06-04T14:37:30.785 & 18.43 & 6.91 & 0.874 & $1.9 \pm 0.7$ \\
\hline 7 & 4560010101 & 2021-06-04T14:43:50.878 & 15.12 & 11.32 & 0.316 & $4.0 \pm 1.0$ \\
\hline 8 & 4560010102 & 2021-06-05T05:57:03.457 & 49.87 & 6.19 & 0.449 & $2.1 \pm 0.7$ \\
\hline $9^{\mathrm{a}}$ & 4560010102 & 2021-06-05T13:55:42.156 & 15.91 & 5.67 & 0.504 & $1.3 \pm 0.6$ \\
\hline 10 & 4560010102 & 2021-06-05T15:42:02.189 & 14.03 & 6.67 & 0.910 & $1.9 \pm 0.7$ \\
\hline 11 & 4560010102 & 2021-06-05T15:43:00.342 & 16.03 & 7.43 & 0.971 & $1.9 \pm 0.7$ \\
\hline 12 & 4560010102 & 2021-06-05T17:12:27.003 & 1.35 & 5.50 & 0.919 & $0.8 \pm 0.5$ \\
\hline 13 & 4560010102 & 2021-06-05T21:55:06.519 & 98.82 & 6.41 & 0.391 & $2.6 \pm 0.8$ \\
\hline 14 & 4560010102 & 2021-06-05T23:31:46.102 & 34.26 & 6.50 & 0.454 & $2.4 \pm 0.8$ \\
\hline $15^{\mathrm{b}}$ & 4560010103 & 2021-06-06T05:36:42.18 & 0.27 & 10.62 & 0.523 & $2.1 \pm 0.7$ \\
\hline 16 & 4560010105 & 2021-06-08T11:19:42.895 & 9.55 & 7.63 & 0.285 & $1.9 \pm 0.7$ \\
\hline 17 & 4560010105 & 2021-06-08T11:23:17.712 & 3.20 & 5.95 & 0.922 & $1.1 \pm 0.5$ \\
\hline 18 & 4560010201 & 2021-06-09T05:51:19.191 & 19.64 & 10.85 & 0.299 & $4.2 \pm 1.1$ \\
\hline 19 & 4560010201 & 2021-06-09Т07:24:11.030 & 7.34 & 6.13 & 0.375 & $1.3 \pm 0.6$ \\
\hline 20 & 4560010201 & 2021-06-09T10:34:21.954 & 7.21 & 6.50 & 0.747 & $1.3 \pm 0.6$ \\
\hline 21 & 4560010201 & 2021-06-09T13:41:17.195 & 25.52 & 8.77 & 0.438 & $3.2 \pm 0.9$ \\
\hline 22 & 4560010201 & 2021-06-09T13:42:48.797 & 19.87 & 6.18 & 0.162 & $1.6 \pm 0.6$ \\
\hline 23 & 4560010202 & 2021-06-10T02:00:03.424 & 14.08 & 6.89 & 0.687 & $1.9 \pm 0.7$ \\
\hline 24 & 4560010202 & 2021-06-10T05:05:18.924 & 21.26 & 6.24 & 0.540 & $1.9 \pm 0.7$ \\
\hline 25 & 4560010301 & 2021-06-11T10:39:52.520 & 6.94 & 6.11 & 0.570 & $1.3 \pm 0.6$ \\
\hline 26 & 4560010602 & 2021-06-17T02:51:59.547 & 6.71 & 16.58 & 0.542 & $6.6 \pm 1.3$ \\
\hline 27 & 4560010602 & 2021-06-17T02:53:19.142 & 54.05 & 19.37 & 0.162 & $13.2 \pm 1.9$ \\
\hline 28 & 4560010602 & 2021-06-17T02:59:19.487 & 72.30 & 7.00 & 0.493 & $2.6 \pm 0.8$ \\
\hline 29 & 4560010701 & 2021-06-19T04:33:05.105 & 7.04 & 7.19 & 0.028 & $1.6 \pm 0.6$ \\
\hline 30 & 4560010701 & 2021-06-19T04:40:15.346 & 7.71 & 7.19 & 0.457 & $1.9 \pm 0.7$ \\
\hline 31 & 4560010701 & 2021-06-19Т06:07:18.896 & 23.03 & 9.82 & 0.305 & $3.4 \pm 1.0$ \\
\hline $32^{\mathrm{c}}$ & 4560010801 & 2021-06-21T17:04:36.952 & 8.54 & 20.58 & 0.664 & $10.8 \pm 1.7$ \\
\hline $32-1^{\mathrm{d}}$ & 4560010801 & 2021-06-21T17:04:36.960 & 21.55 & 6.25 & 0.668 & $2.6 \pm 0.8$ \\
\hline 33 & 4560010801 & 2021-06-21T23:05:26.979 & 17.84 & 6.39 & 0.767 & $1.6 \pm 0.6$ \\
\hline 34 & 4560010801 & 2021-06-21T23:06:53.759 & 35.45 & 8.78 & 0.244 & $3.2 \pm 0.9$ \\
\hline 35 & 4560010802 & 2021-06-22T00:35:18.974 & 12.50 & 8.94 & 0.228 & $2.4 \pm 0.8$ \\
\hline 36 & 4560010802 & 2021-06-22T00:45:29.330 & 15.47 & 8.55 & 0.307 & $2.6 \pm 0.8$ \\
\hline 37 & 4560010802 & 2021-06-22T02:20:44.171 & 32.71 & 8.16 & 0.385 & $2.9 \pm 0.9$ \\
\hline 38 & 4560010802 & 2021-06-22T03:55:16.474 & 30.36 & 10.37 & 0.445 & $4.0 \pm 1.0$ \\
\hline 39 & 4560010802 & 2021-06-22Т08:26:59.567 & 6.15 & 12.71 & 0.746 & $4.0 \pm 1.0$ \\
\hline 40 & 4560010802 & 2021-06-22T08:29:34.911 & 49.79 & 19.15 & 0.984 & $10.6 \pm 1.7$ \\
\hline 41 & 4560010802 & 2021-06-22T10:00:07.220 & 15.05 & 8.79 & 0.882 & $2.6 \pm 0.8$ \\
\hline 42 & 4560010902 & 2021-06-24T06:50:27.424 & 5.97 & 5.68 & 0.654 & $1.1 \pm 0.5$ \\
\hline 43 & 4560011001 & 2021-06-26T00:52:34.199 & 12.48 & 9.24 & 0.167 & $2.6 \pm 0.8$ \\
\hline 44 & 4560011301 & 2021-07-01T21:40:50.682 & 211.22 & 7.86 & 0.284 & $4.0 \mathrm{pm} 1.0$ \\
\hline 45 & 4560011301 & $2021-07-01 T 23: 21: 28.122$ & 5.28 & 12.89 & 0.860 & $4.2 \pm 1.1$ \\
\hline
\end{tabular}

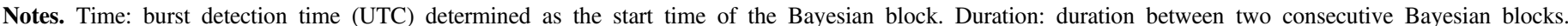

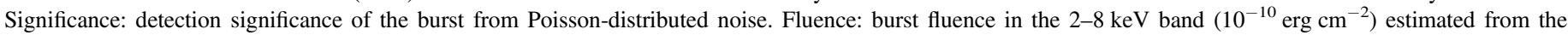

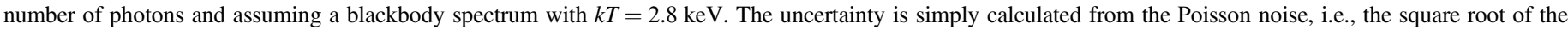
number of photons.

a This burst was simultaneously observed with the second observation of DSN (Table A4).

b This burst was simultaneously observed with the third observation of DSN (Table A4).

c This burst was simultaneously observed with Swift BAT (burst 5 in Table B1).

d This candidate is the tail of burst 27 .

turnover, which may be caused by the detection limit of NICER. We fit the tail and found a power-law index of $-1.5 \pm 0.2$. The uncertainty is estimated from $10^{4}$ times of Monte Carlo simulations. In each run, we simulate 45 bursts, and the number of photons of each burst is simulated from Poisson-distributed noise with a mean value the same as the observed one. Then we rebin the distribution and obtain the power-law index with data points with a fluence higher than $2 \times 10^{-10} \mathrm{erg} \mathrm{cm}^{-2}$.

A burst (burst number 5 in Table B1 and 32 in Table B2) is simultaneously detected with Swift BAT and NICER. The light 
curves of this simultaneous event are shown in Figure B2(a) and (b). We extracted the broadband X-ray spectrum and fit it with an absorbed power law and set $N_{\mathrm{H}}=8.72 \times 10^{22} \mathrm{~cm}^{-2}$ (see Figures B2(c) and (d)). The spectrum can be fitted by two blackbodies, although the normalization cannot be well constrained. The soft one has a temperature of $k T=2.5_{-0.7}^{+3.3} \mathrm{keV}$, which is set to be 0.37 times the temperature of the hard component (Nakagawa et al. 2009). On the other hand, the spectrum can also be well fitted by an absorbed blackbody with $k T=5.3 \pm 0.8 \mathrm{keV}$ and a radius of $7.0 \pm 1.6 \mathrm{~km}$ at $10 \mathrm{kpc}$.

\section{Appendix C \\ Comparison with Previous Magnetar Outbursts}

Tables $\mathrm{C} 1$ and $\mathrm{C} 2$ summarize the properties of previous magnetar outbursts.

Table C1

A List of X-Ray Outbursts of Radio-loud Transient Magnetars

\begin{tabular}{|c|c|c|c|c|c|c|c|}
\hline Source & State & $\begin{array}{c}L_{\mathrm{x}} \\
\left(\mathrm{erg} \mathrm{s}^{-1}\right)\end{array}$ & $\begin{array}{c}L_{\mathrm{sd}} \\
\left(\mathrm{erg} \mathrm{s}^{-1}\right)\end{array}$ & $\begin{array}{l}\text { Distance } \\
(\mathrm{kpc})\end{array}$ & PF & $\begin{array}{l}\text { Energy } \\
(\mathrm{keV})\end{array}$ & References \\
\hline \multirow[t]{3}{*}{ 1E $1547.0-5408$} & Quiescent & $2.2 \times 10^{33}$ & $2.11 \times 10^{35}$ & 4.5 & 0.205 & $0.5-2$ & 1,2 \\
\hline & Outburst (2008) & $2.3 \times 10^{35}$ & & & 0.26 & $0.5-10$ & 1,3 \\
\hline & Outburst (2009) & $5.0 \times 10^{35}$ & & & 0.13 & $0.5-3$ & 1,4 \\
\hline \multirow[t]{3}{*}{ XTE J1810-197 } & Quiescent & $2.5 \times 10^{34}$ & $1.80 \times 10^{33}$ & 3.5 & 0.212 & $0.5-2$ & 1,2 \\
\hline & Outburst (2003) & $1.7 \times 10^{35}$ & & & 0.43 & $1-1.5$ & 1,5 \\
\hline & Outburst (2018) & $2.5 \times 10^{35}$ & & & 0.27 & $0.5-2$ & 6,7 \\
\hline \multirow[t]{2}{*}{ PSR J1622-4950 } & Quiescent & $<7.7 \times 10^{32}$ & $8.27 \times 10^{33}$ & 9 & & & 8 \\
\hline & Outburst (2017) & $1.5 \times 10^{35}$ & & & 0.04 & $0.3-6$ & 8 \\
\hline \multirow[t]{2}{*}{ SGR $1745-1900$} & Quiescent & $4.7 \times 10^{33}$ & $1.02 \times 10^{34}$ & 8.3 & 0.26 & $0.5-7$ & 2,9 \\
\hline & Outburst (2013) & $6.8 \times 10^{35}$ & & & 0.45 & $0.3-3.5$ & 1,10 \\
\hline \multirow[t]{2}{*}{ Swift J1818.0-1607 } & Quiescent & $<1.7 \times 10^{34}$ & $1.40 \times 10^{36}$ & 6.5 & & & 11 \\
\hline & Outburst (2020) & $1.9 \times 10^{35}$ & & & 0.52 & $1-3$ & 11,12 \\
\hline \multirow[t]{3}{*}{ SGR $1935+2154$} & Quiescent & $1.1 \times 10^{34}$ & $1.65 \times 10^{34}$ & 9 & 0.1 & $0.5-2$ & 2,13 \\
\hline & Outburst (2014) & $2.5 \times 10^{34}$ & & & 0.17 & $0.5-1.5$ & 1,14 \\
\hline & Outburst (2020) & $1.6 \times 10^{34}$ & & & 0.14 & $0.7-3$ & 13,15 \\
\hline \multirow[t]{2}{*}{ PSR J1119-6127 } & Quiescent & $5.7 \times 10^{32}$ & $2.33 \times 10^{36}$ & 8.4 & 0.74 & $0.5-2$ & 1,16 \\
\hline & Outburst (2016) & $3.7 \times 10^{35}$ & & & 0.67 & $0.7-3$ & 1,17 \\
\hline
\end{tabular}

Note. $L_{\mathrm{x}}$ : observed X-ray luminosity $(0.3-10 \mathrm{keV})$ assuming the distance in the right column. $L_{\mathrm{sd}}$ : spin-down luminosity. PF: X-ray PF defined in the energy band in the right column.

References. 1. Coti Zelati et al. (2018); 2. Hu et al. (2019); 3. Israel et al. (2010); 4. Bernardini et al. (2011); 5. Gotthelf et al. (2004); 6. Pearlman et al. (2020); 7. Borghese et al. (2021); 8. Camilo et al. (2018); 9. Rea et al. (2020); 10. Coti Zelati et al. (2015); 11. Hu et al. (2020); 12. Esposito et al. (2020); 13. Borghese et al. (2020); 14. Israel et al. (2016); 15. Göğüş et al. (2020); 16. Gonzalez et al. (2005); 17. Archibald et al. (2018). 
Table C2

A List of X-Ray Outbursts of Radio-quiet Transient Magnetars

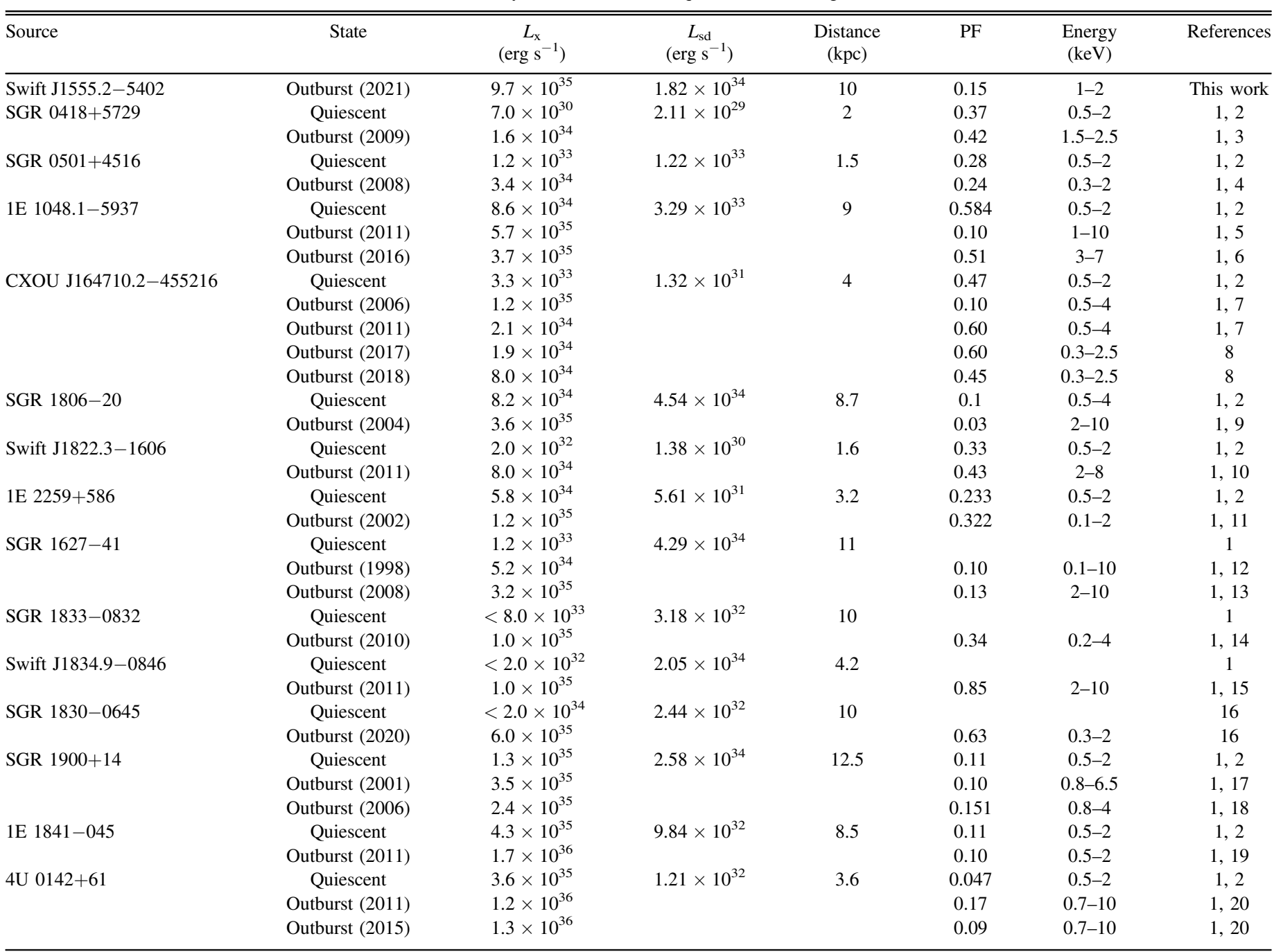

Note. Definitions of the columns are the same as in Table C1.

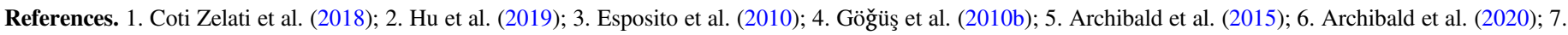

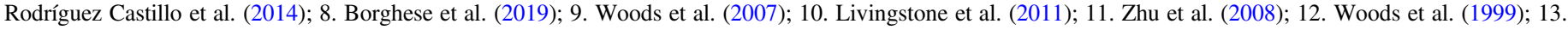

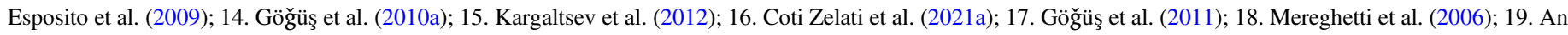
et al. (2013); 20. Archibald et al. (2017).

\section{ORCID iDs}

Teruaki Enoto (iD https://orcid.org/0000-0003-1244-3100

Mason Ng (i) https://orcid.org/0000-0002-0940-6563

Chin-Ping Hu (D) https://orcid.org/0000-0001-8551-2002

Tolga Güver (iD https://orcid.org/0000-0002-3531-9842

Gaurava K. Jaisawal (i) https://orcid.org/0000-0002-

6789-2723

Brendan O'Connor (i) https://orcid.org/0000-0002-9700-0036

Ersin Göğüş (DD https://orcid.org/0000-0002-5274-6790

Shota Kisaka (1) https://orcid.org/0000-0002-2498-1937

Zorawar Wadiasingh (iD https://orcid.org/0000-0002-

9249-0515

Walid A. Majid (iD https://orcid.org/0000-0002-4694-4221

Aaron B. Pearlman (10 https://orcid.org/0000-0002-8912-0732

Karishma Bansal (D) https://orcid.org/0000-0002-7418-7862

Harsha Blumer (ib https://orcid.org/0000-0003-4046-884X

Deepto Chakrabarty (iD https://orcid.org/0000-0001-

8804-8946
Keith Gendreau (1D https://orcid.org/0000-0001-7115-2819

Wynn C. G. Ho (ib https://orcid.org/0000-0002-6089-6836

Chryssa Kouveliotou (iD https://orcid.org/0000-00031443-593X

Paul S. Ray (1D https://orcid.org/0000-0002-5297-5278

Tod E. Strohmayer (i) https://orcid.org/0000-0001-7681-5845

George Younes (iD https://orcid.org/0000-0002-7991-028X

David M. Palmer (iD https://orcid.org/0000-0001-7128-0802

Takanori Sakamoto (iD https://orcid.org/0000-0001-6276-6616

Takuya Akahori (D) https://orcid.org/0000-0001-9399-5331

Sujin Eie (i) https://orcid.org/0000-0003-0340-0651

\section{References}

Agarwal, D., Aggarwal, K., Burke-Spolaor, S., Lorimer, D. R., \& Garver-Daniels, N. 2020, MNRAS, 497, 1661

An, H., Hascoët, R., Kaspi, V. M., et al. 2013, ApJ, 779, 163

Archibald, R. F., Kaspi, V. M., Ng, C. Y., et al. 2015, ApJ, 800, 33

Archibald, R. F., Kaspi, V. M., Scholz, P., et al. 2017, ApJ, 834, 163 
Archibald, R. F., Kaspi, V. M., Tendulkar, S. P., \& Scholz, P. 2018, ApJ, 869,180

Archibald, R. F., Scholz, P., Kaspi, V. M., Tendulkar, S. P., \& Beardmore, A. P. 2020, ApJ, 889, 160

Arnaud, K. A. 1996, in ASP Conf. Ser. 101, Astronomical Data Analysis Software and Systems V, ed. G. H. Jacoby \& J. Barnes (San Francisco, CA: ASP), 17

Arons, J., \& Scharlemann, E. T. 1979, ApJ, 231, 854

Arzoumanian, Z., Chernoff, D. F., \& Cordes, J. M. 2002, ApJ, 568, 289

Astropy Collaboration, Robitaille, T. P., Tollerud, E. J., et al. 2013, A\&A, 558, A33

Bansal, K., Pearlman, A. B., Majid, W. A., et al. 2021, ATel, 14678, 1

Baring, M. G., \& Harding, A. K. 2001, ApJ, 547, 929

Barthelmy, S. D., Barbier, L. M., Cummings, J. R., et al. 2005, SSRv, 120, 143

Beloborodov, A. M., \& Li, X. 2016, ApJ, 833, 261

Bernardini, F., Israel, G. L., Stella, L., et al. 2011, A\&A, 529, A19

Bildsten, L., Chakrabarty, D., Chiu, J., et al. 1997, ApJS, 113, 367

Blumer, H., Safi-Harb, S., Borghese, A., et al. 2021, arXiv:2106.12018

Bochenek, C. D., Ravi, V., Belov, K. V., et al. 2020, Natur, 587, 59

Borghese, A., Coti Zelati, F., Rea, N., et al. 2020, ApJL, 902, L2

Borghese, A., Rea, N., Turolla, R., et al. 2019, MNRAS, 484, 2931

Borghese, A., Rea, N., Turolla, R., et al. 2021, MNRAS, 504, 5244

Buccheri, R., Bennett, K., Bignami, G. F., et al. 1983, A\&A, 128, 245

Burgay, M., Pilia, M., Possenti, A., et al. 2021, ATel, 14680, 1

Burrows, D. N., Hill, J. E., Nousek, J. A., et al. 2005, SSRv, 120, 165

Camilo, F., Ransom, S. M., Halpern, J. P., \& Reynolds, J. 2007a, ApJL, 666, L93

Camilo, F., Ransom, S. M., Peñalver, J., et al. 2007b, ApJ, 669, 561

Camilo, F., Reynolds, J., Johnston, S., Halpern, J. P., \& Ransom, S. M. 2008 , ApJ, 679, 681

Camilo, F., Scholz, P., Serylak, M., et al. 2018, ApJ, 856, 180

CHIME/FRB Collaboration, Andersen, B. C., Bandura, K. M., et al. 2020, Natur, 587, 54

Coti Zelati, F., Borghese, A., Israel, G. L., et al. 2021a, ApJL, 907, L34

Coti Zelati, F., Borghese, A., Rea, N., et al. 2021b, ATel, 14674, 1

Coti Zelati, F., Rea, N., Papitto, A., et al. 2015, MNRAS, 449, 2685

Coti Zelati, F., Rea, N., Pons, J. A., Campana, S., \& Esposito, P. 2018, MNRAS, 474, 961

Dib, R., \& Kaspi, V. M. 2014, ApJ, 784, 37

Dib, R., Kaspi, V. M., Scholz, P., \& Gavriil, F. P. 2012, ApJ, 748, 3

Enoto, T., Kisaka, S., \& Shibata, S. 2019, RPPh, 82, 106901

Enoto, T., Nakazawa, K., Makishima, K., et al. 2010a, ApJL, 722, L162

Enoto, T., Nakazawa, K., Makishima, K., et al. 2010b, PASJ, 62, 475

Enoto, T., Shibata, S., Kitaguchi, T., et al. 2017, ApJS, 231, 8

Esposito, P., Burgay, M., Possenti, A., et al. 2009, MNRAS, 399, L44

Esposito, P., Israel, G. L., Turolla, R., et al. 2010, MNRAS, 405, 1787

Esposito, P., Rea, N., Borghese, A., et al. 2020, ApJL, 896, L30

Evans, P. A. 2021, ATel, 14675, 1

Gehrels, N., Chincarini, G., Giommi, P., et al. 2004, ApJ, 611, 1005

Gelfand, J. D., \& Gaensler, B. M. 2007, ApJ, 667, 1111

Gendreau, K. C., Arzoumanian, Z., Adkins, P. W., et al. 2016, Proc. SPIE, 9905, 99051H

Goldreich, P., \& Julian, W. H. 1969, ApJ, 157, 869

Gonzalez, M. E., Kaspi, V. M., Camilo, F., Gaensler, B. M., \& Pivovaroff, M. J. 2005, ApJ, 630, 489

Gotthelf, E. V., Halpern, J. P., Buxton, M., \& Bailyn, C. 2004, ApJ, 605, 368 Göğüş, E., Baring, M. G., Kouveliotou, C., et al. 2020, ApJL, 905, L31

Göğüş, E. 2014, AN, 335, 296

Göğüş, E., Cusumano, G., Levan, A. J., et al. 2010a, ApJ, 718, 331

Göğüs, E., Güver, T., Özel, F., Eichler, D., \& Kouveliotou, C. 2011, ApJ, 728,160

Göğüş, E., Woods, P. M., Kouveliotou, C., et al. 2010b, ApJ, 722, 899

Green, D. A. 2019, JApA, 40, 36

Harrison, F. A., Craig, W. W., Christensen, F. E., et al. 2013, ApJ, 770, 103

Ho, W. C. G. 2013, MNRAS, 429, 113

Hu, C.-P., Begiçarslan, B., Güver, T., et al. 2020, ApJ, 902, 1

Hu, C.-P., Ng, C. Y., \& Ho, W. C. G. 2019, MNRAS, 485, 4274

Huang, Z.-P., Yan, Z., Shen, Z.-Q., et al. 2021, MNRAS, 505, 1311

Israel, G. L., Esposito, P., Rea, N., et al. 2010, MNRAS, 408, 1387

Israel, G. L., Esposito, P., Rea, N., et al. 2016, MNRAS, 457, 3448

Israel, G. L., Zelati, F. C., Borghese, A., et al. 2021, ATel, 14685, 1

Kargaltsev, O., Kouveliotou, C., Pavlov, G. G., et al. 2012, ApJ, 748, 26

Kaspi, V. M., \& Beloborodov, A. M. 2017, ARA\&A, 55, 261

Kouveliotou, C., Dieters, S., Strohmayer, T., et al. 1998, Natur, 393, 235
Kuiper, L., Hermsen, W., den Hartog, P. R., \& Collmar, W. 2006, ApJ, 645, 556

Lazarus, P., Kaspi, V. M., Champion, D. J., Hessels, J. W. T., \& Dib, R. 2012, ApJ, 744, 97

Levin, L., Bailes, M., Bates, S., et al. 2010, ApJL, 721, L33

Levin, L., Bailes, M., Bates, S. D., et al. 2012, MNRAS, 422, 2489

Levin, L., Lyne, A. G., Desvignes, G., et al. 2019, MNRAS, 488, 5251

Li, C. K., Lin, L., Xiong, S. L., et al. 2021, NatAs, 5, 378

Lien, A., Sakamoto, T., Barthelmy, S. D., et al. 2016, ApJ, 829, 7

Livingstone, M. A., Scholz, P., Kaspi, V. M., Ng, C. Y., \& Gavriil, F. P. 2011, ApJL, 743, L38

Lower, M. E., Johnston, S., Shannon, R. M., Bailes, M., \& Camilo, F. 2021, MNRAS, 502, 127

Lower, M. E., Shannon, R. M., Johnston, S., \& Bailes, M. 2020, ApJL, 896, L37

Luo, J., Ransom, S., Demorest, P., et al. 2021, ApJ, 911, 45

Lyne, A. G., \& Manchester, R. N. 1988, MNRAS, 234, 477

Majid, W. A., Pearlman, A. B., Dobreva, T., et al. 2017, ApJL, 834, L2

Manchester, R. N., Hobbs, G. B., Teoh, A., \& Hobbs, M. 2005, AJ, 129, 1993

Mereghetti, S. 2008, A\&ARv, 15, 225

Mereghetti, S., Esposito, P., Tiengo, A., et al. 2006, ApJ, 653, 1423

Mereghetti, S., Savchenko, V., Ferrigno, C., et al. 2020, ApJL, 898, L29

Nakagawa, Y. E., Yoshida, A., Yamaoka, K., \& Shibazaki, N. 2009, PASJ, 61,109

Nasa High Energy Astrophysics Science Archive Research Center (Heasarc) 2014, HEAsoft: Unified Release of FTOOLS and XANADU, Astrophysics Source Code Library, ascl:1408.004

Ng, C. Y., Kaspi, V. M., Ho, W. C. G., et al. 2012, ApJ, 761, 65

Ng, M., Hu, C. P., Enoto, T., et al. 2021, ATel, 14684, 1

Olausen, S. A., \& Kaspi, V. M. 2014, ApJS, 212, 6

Palmer, D. 2021, ATel, 14690, 1

Palmer, D. M., Evans, P. A., Kuin, N. P. M., Page, K. L. \& Swift Team 2021, GCN Circ., 30120, 1, https://gcn.gsfc.nasa.gov/other/SGRJ1555.25402.gcn 3

Pearlman, A. B., Majid, W. A., \& Prince, T. A. 2019, AdAst, 2019, 6325183

Pearlman, A. B., Majid, W. A., Prince, T. A., et al. 2020, arXiv:2005.08410

Pearlman, A. B., Majid, W. A., Prince, T. A., Kocz, J., \& Horiuchi, S. 2018, ApJ, 866, 160

Pennucci, T. T., Possenti, A., Esposito, P., et al. 2015, ApJ, 808, 81

Perna, R., \& Pons, J. A. 2011, ApJL, 727, L51

Potekhin, A. Y., Pons, J. A., \& Page, D. 2015, SSRv, 191, 239

Price-Whelan, A. M., Sipőcz, B. M., Günther, H. M., et al. 2018, AJ, 156, 123

Rankin, J. M. 1993, ApJ, 405, 285

Ransom, S. M., Eikenberry, S. S., \& Middleditch, J. 2002, AJ, 124, 1788

Rea, N., Coti Zelati, F., Viganò, D., et al. 2020, ApJ, 894, 159

Remillard, R. A., Loewenstein, M., Steiner, J. F., et al. 2021, arXiv:2105. 09901

Reynolds, M. T., Miller, J. M., Maitra, D., et al. 2013, ATel, 5200, 1

Ridnaia, A., Svinkin, D., Frederiks, D., et al. 2021, NatAs, 5, 372

Rodríguez Castillo, G. A., Israel, G. L., Esposito, P., et al. 2014, MNRAS, 441, 1305

Ruderman, M. A., \& Sutherland, P. G. 1975, ApJ, 196, 51

Scargle, J. D., Norris, J. P., Jackson, B., \& Chiang, J. 2013, ApJ, 764, 167

Shannon, R. M., \& Johnston, S. 2013, MNRAS, 435, L29

Singh, S., \& Roy, J. 2021, ATel, 14799, 1

Standish, E. M. 1998, JPL Planetary and Lunar Ephemerides, DE405/LE405, JPL Interoffice Memo 312.F-98-048, (Pasadena: NASA Jet Propulsion Laboratory)

Sturrock, P. A. 1971, ApJ, 164, 529

Tavani, M., Casentini, C., Ursi, A., et al. 2021, NatAs, 5, 401

Thompson, C., \& Duncan, R. C. 1995, MNRAS, 275, 255

Thompson, C., \& Duncan, R. C. 1996, ApJ, 473, 322

Tiengo, A., Vianello, G., Esposito, P., et al. 2010, ApJ, 710, 227

Wadiasingh, Z., Baring, M. G., Gonthier, P. L., \& Harding, A. K. 2018, ApJ, 854, 98

Wadiasingh, Z., \& Timokhin, A. 2019, ApJ, 879, 4

Wilms, J., Allen, A., \& McCray, R. 2000, ApJ, 542, 914

Woods, P. M., Kaspi, V. M., Thompson, C., et al. 2004, ApJ, 605, 378

Woods, P. M., Kouveliotou, C., Finger, M. H., et al. 2007, ApJ, 654, 470

Woods, P. M., Kouveliotou, C., van Paradijs, J., et al. 1999, ApJL, 519, L139

Younes, G., Baring, M. G., Kouveliotou, C., et al. 2017a, ApJ, 851, 17

Younes, G., Kouveliotou, C., Jaodand, A., et al. 2017b, ApJ, 847, 85

Zhu, W., Kaspi, V. M., Dib, R., et al. 2008, ApJ, 686, 520 